\title{
Capturing the two-way hydromechanical coupling effect on fluid-driven fracture in a dual-graph lattice beam model
}

\author{
Ole Ivar Ulven ${ }^{1} \mathbb{\text { | }}$ WaiChing Sun ${ }^{2}$
}

\author{
${ }^{1}$ Physics of Geological Processes (PGP), \\ Department of Geosciences, University of \\ Oslo, P.O. Box 1047 Blindern Oslo, 0316, \\ Norway \\ ${ }^{2}$ Department of Civil Engineering and \\ Engineering Mechanics, The Fu \\ Foundation School of Engineering and \\ Applied Science, Columbia University in \\ the City of New York, New York, 10027, \\ NY, USA

\section{Correspondence} \\ Ole Ivar Ulven, Physics of Geological \\ Processes (PGP), Department of \\ Geosciences, University of Oslo, P.O. Box \\ 1047 Blindern, 0316 Oslo, Norway. \\ Email: o.i.ulven@fys.uio.no

\section{Funding information} \\ Norwegian Research Council, \\ Grant/Award Number: 231621; Earth \\ Materials and Processes program from the \\ US Army Research Office, Grant/Award \\ Number: W911NF-15-1-0442 and \\ W911NF-15-1-0581; Dynamic Materials \\ and Interactions Program from the Air \\ Force Office of Scientific Research, \\ Grant/Award Number: FA9550-17-1-0169; \\ Nuclear Energy University Program from \\ Department of Energy, Grant/Award \\ Number: DE-NE0008534; Earth Science \\ Program, Grant/Award Number: \\ EAR-1520732
}

\begin{abstract}
Summary
Fluid-driven fractures of brittle rock is simulated via a dual-graph lattice model. The new discrete hydromechanical model incorporates a two-way coupling mechanism between the discrete element model and the flow network. By adopting an operator-split algorithm, the coupling model is able to replicate the transient poroelasticity coupling mechanism and the resultant Mandel-Cryer hydromechanical coupling effect in a discrete mechanics framework. As crack propagation, coalescence and branching are all path-dependent and irreversible processes, capturing this transient coupling effect is important for capturing the essence of the fluid-driven fracture in simulations. Injection simulations indicate that the onset and propagation of fractures is highly sensitive to the ratio between the injection rate and the effective permeability. Furthermore, we show that in a permeable rock, the borehole breakdown pressure, the pressure at which fractures start to grow from the borehole, depends on both the given ratio between injection rate and permeability and the Biot coefficient.
\end{abstract}

\section{KEYWORDS}

dual graph, fluid-driven fracture, lattice method, operator split, poroelasticity

\section{1 | INTRODUCTION}

Onset and propagation of fractures in geological materials is often induced or influenced by the pore-fluid flowing inside the pores or preexisting microvoids and cracks. This hydromechanical coupling process has important implications for hydraulic fracturing for oil and gas recovery, management of nuclear waste disposals, and the geological storage of carbon dioxide.

Of special interest is in many cases the study of fracture formation around a borehole during fluid injection. This is commonly used in stimulated production of hydrocarbons and to determine the in situ stress field in a rock mass, but also has its application in volcanic systems, where magma injected in a dyke might cause fracture formation around the dyke. This type of processes, however, represent significant challenges for numerical studies. 
Previous attempts on capturing the fluid-driven fracture processes can be categorized into 2 classes of approaches. The first is the continuum modeling approach in which the fluid-infiltrated geological materials are idealized as a multiphase continuum mixture. ${ }^{1-3}$ The fracture surfaces are then captured by embedded strong discontinuities ${ }^{4-12}$ or represented by a level set function ${ }^{13-16}$ in the continuum body. A force-separation or cohesive law is used to replicate the path-dependent responses of the brittle solid, while the hydraulic properties of the fractures are captured via the so-called cubic law. For example, the pioneer work in Ingraffea and Saouma ${ }^{17}$ has attempted to simulate the opening of a crack in a fully saturated porous media by coupling a 2D finite element model with a 1D finite difference model (FDM) to simulate the hydromechanical coupling effect when the crack mouth is opened by the injected fluid. Recent works ${ }^{4,18,19}$ either introduce enrichment functions inside a continuous finite element model or insert interface elements along the element edges to represent fractures as displacement jumps. In both cases, the modeling of crack nucleation and propagation is challenging, as the enhanced continuum approach requires constant modification of the mesh topology to accommodate the crack growth. ${ }^{20}$ Furthermore, any attempt to re-mesh a domain occupied by a path-dependent material will require projection of history-dependent internal variables. ${ }^{21,22}$ Another feasible continuum approach that becomes increasingly popular is the phase field model. ${ }^{13,15,16}$ The key idea of the phase field model is to represent the fracture not by a displacement jump, but by a scalar implicit function that indicates the status of the material in the space. As a result, crack nucleation, coalescence, and branching can be captured without introducing any special mesh treatment. Nevertheless, the phase field approach also requires a sufficiently fine mesh size typically in the order of the characteristic length. This characteristic length, in the case of phase field models, defines the size of the regularized damage zone and converges to a discrete fracture only when this length scale approaches zero. ${ }^{15,16,23}$ Therefore, the demand on computational power is significant.

As an alternative to the continuum approach, discrete and lattice models have long been used to model the mechanical and hydromechanical responses of geological materials. ${ }^{24-29}$ An important upshot of the discrete approaches is that they offer a simpler way to represent fractures and crack growth. Since the discrete models idealize the material as networks of particles or points connected by a finite number of lattice elements or force chains that distribute force and moment, the onset and propagation of cracks can be replicated numerically by incorporating damage, softening, or de-bonding constitutive laws in the lattices. A promising possibility is to embed a discrete model inside a larger domain described by a continuum based model in a hybrid approach. ${ }^{30}$ For two-dimensional problems, the discrete model may also extend to incorporate a deformation-diffusion coupling effect. ${ }^{26,31-33}$ In particular, Grassl et a ${ }^{26}$ use the dual-graph concept from graph theory to generate a flow network that evolves in accordance with the deformation of the solid network. This dual-graph idea has also been used to analyze the topology of void space for granular materials. ${ }^{34-37}$ The innovative approach in Grassl et al ${ }^{26}$ uses the dual-graph of the contact network to represent the pore space. By assigning constant hydraulic conductivity to the edges of the dual graph and assuming that the fluid in the void is already in steady state, fluid transport in the void space can be captured by solving the Poisson equation in the discrete dual graph and provide feedback to the solid solver.

The aim of this paper is twofold. Firstly, we introduce a two-way coupled hydromechanical model, and secondly, we apply this model to fracture formation around boreholes and provide new insights on borehole breakdown. We adopt the same dual-graph concept as in Grassl et $\mathrm{al}^{26}$ to establish the discrete element model (DEM)-network coupling model. However, the major departure is the introduction of a two-way hydromechanical coupling effect between the discrete fluid and solid solver. This treatment essentially relaxes the steady-state assumption. We apply this model to a borehole injection setting and study differences in fracture formation for different rock permeability, Biot coefficient, fluid injection rate, and fracture permeability. Further, we show that the borehole breakdown pressure for a permeable rock depends strongly on the studied parameters. Finally, we show that for sufficiently high grid resolution, the fracture formation around a borehole is not significantly changed by increasing the resolution.

As shown in the analytical solutions and numerical examples in this paper, the two-way coupling has particularly important implications on capturing the fracture behavior for low permeability media subjected to high injection rate. Since the fracture process is path and history dependent, capturing the two-way coupling of the evolution of fractures and hydraulic responses is of ultimate importance in simulating the development of flow conduits. To the best knowledge of the authors, this is the first work that uses an iterative two-way coupling DEM-network model to simulate hydraulic fractures.

The rest of this paper is organized as follows. Section 2 introduces the basic equations describing the process and briefly outlines a simplified 1D FDM for the coupled process, before presenting in detail the sequentially iterative discrete model used to simulate the transient hydromechanical coupling processes. Section 3 presents the benchmark study that verify the implementation of the model and the results of a series of numerical simulations of injection-induced crack growth, followed by a discussion of borehole fracture results and comparison of the proposed model with previous work, and the conclusion section that summarizes the major findings. 
As for notations and symbols, boldfaced letters denote tensors; the symbol "." denotes a single contraction of adjacent indices of 2 tensors (eg, $\boldsymbol{a} \cdot \boldsymbol{b}=a_{i} b_{i}$ or $\boldsymbol{c} \cdot \boldsymbol{d}=c_{i j} d_{j k}$ ); the symbol " $\otimes$ " denotes a juxtaposition of 2 vectors (eg, $\left.\boldsymbol{a} \otimes \boldsymbol{b}=a_{i} b_{j}\right)$ or 2 symmetric second-order tensors (eg, $\left.(\boldsymbol{\alpha} \otimes \boldsymbol{\beta})=\alpha_{i j} \beta_{k l}\right)$. As for sign conventions, we consider the direction of the tensile stress and dilative pressure as positive.

\section{2 | METHODS}

The models describe the coupled process of fluid injection into a fluid saturated solid and mechanical deformation of the solid due to the internal fluid pressure. This is achieved by applying Biot theory for the behavior of porous solids, ${ }^{38,39}$ where the total stress $\sigma$ in the mechanical model is made up of an effective stress $\sigma_{m}$ carried by the porous solid and a stress $\sigma_{f}$ caused by the fluid pressure in the pores:

$$
\sigma=\sigma_{m}+b \sigma_{f}
$$

Here, $b$ is Biot coefficient. When gravity and surface forces are ignored, fluid transport in the solid is described by ${ }^{39}$

$$
\frac{1}{M} \frac{\partial p}{\partial t}-\frac{k}{\mu} \nabla^{2} p=-b \frac{\partial \varepsilon_{v}}{\partial t},
$$

where $p$ is the fluid pressure, $M$ is the Biot modulus, $t$ denotes time, $k$ is the permeability, $\mu$ is the fluid viscosity, and $\varepsilon_{v}$ is the volumetric strain.

Two geometries are chosen, a cylinder for benchmarking towards an analytical solution for point injection ${ }^{2}$ and a thick-walled cylinder for comparison with previous results ${ }^{26}$ and further studies of borehole breakdown. In both cases, no variation in the axial direction is assumed, thus simplifying the geometries to $2 \mathrm{D}$ cross sections. The computational methods applied in this paper are based on a 1D FDM ${ }^{32}$ and a 2D DEM. ${ }^{32,33,40}$ In the previous papers, both models were combined with a reactive transport model describing volume changes. In this paper, we combine the models with a new fluid transport model based on Equation 2. In the following, the mechanical model in DEM is described in detail, a dual grid is introduced, and the fluid transport model is derived. Then the coupling between the mechanical model and the fluid transport model is described. The chapter is concluded by a discussion of model assumptions, relevant parameter ranges, and the scaling behavior of the equations. The one-dimensional FDM is a monolithic solver for a standard discretization of the equations of linear elasticity and the fluid transport model in Equation 2 under the assumption of radial symmetry. The interested reader is referred to Appendix A for details.

\section{1 | The mechanical model}

The DEM we adopted is a beam-based model, which is a modified version of the DEM model introduced by Cundall and Strack. ${ }^{24}$ The underlying idea of the beam-based DEM is to model the solid as a collection of interacting particles that occupy the solid phase of the porous material. The interaction of these particles is then captured by brittle-breaking beam elements that replicates the interparticle breakages in the brittle regime. ${ }^{41-43}$ We use a model where the particles are connected by beams withstanding both tensile and shear stresses, ${ }^{44}$ and the beam behavior can be defined from classical beam theory based on a beam material with unit Young modulus and Poisson ratio $v_{0}$. Note that the lattice of beams can be viewed as a discretized approximation of a generalized continuum that has been widely used for simulating fractures in concrete. In 2 seminal works, ${ }^{42,45}$ the influence of orientation of the beam elements and the size of the beam elements on the crack pattern has been systematically studied. In particular, one interesting finding is that longer beams (and hence lower resolution) tends to give more ductile responses. As the coarser mesh requires more energy to break the longer beams and the breakage events become sporadic, larger deformation may occur in between. It should be noted that mesh bias is not unique for the lattice-beam model, but also occurs in the more conventional embedded strong discontinuity approach in finite elements (eg, Mosler and $\mathrm{Meschke}^{46}$ ). One possible way to reduce-but not completely eliminate-the mesh bias is to use a polygonal lattice (eg, Yip et $\mathrm{al}^{47}$ ) or polygonal finite elements (eg, Leon et $\mathrm{al}^{48}$ ) and choose the mesh size close to the physical length scale or characteristic length. In Grassl et al, ${ }^{26}$ the authors have conducted refinement studies, and the results show that the crack patterns are almost independent of mesh size, although with arbitrary crack orientation due to axisymmetry in the chosen problem, when a sufficient number of beams is used to suppress the artificial ductile behavior and provide sufficient potential crack paths. 

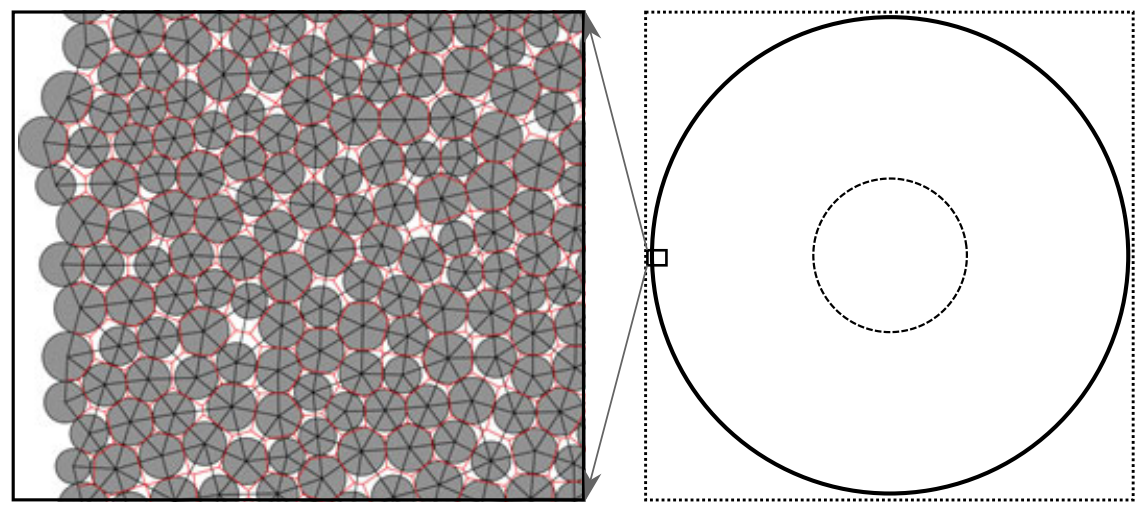

FIGURE 1 The unstructured grid used in this work. From an initially square-shaped grid (dotted line), a circle was cut (solid line). To create a thick-walled cylinder, particles inside the dashed line was removed. The expanded view on the left shows the details of a section of the unstructured grid, with particles shown in gray, beams connecting the particles shown as black lines, and the Voronoi tesselation further introduced in Section 2.2 shown as red lines [Colour figure can be viewed at wileyonlinelibrary.com]

A similar approach is adopted in the present study, where we follow the lines of previous works ${ }^{41,45,49}$ and let each DEM particle represent the averaged properties of several grains below grid resolution. Natural heterogeneities in the grain structure are described by a random distribution of material properties. ${ }^{49}$ In experimental studies, fractures might follow preexisting grain boundaries or cut through grains..$^{50}$ It follows that the grid resolution is limiting the crack density, and sufficient resolution to allow the necessary crack paths is required. Fragmentation even at the resolution of the grid would suggest that the number of potential crack paths is too low and that the material might crack at a subparticle scale if model resolution had been increased. Grid refinement studies in this work (Section 3.6) show that, when the lattice beam is sufficiently short relative to the boundary layer of the pore pressure, the dependence on the lattice beam length is less significant.

To reduce grid-induced bias on fracture directions, an unstructured grid was used (Figure 1). A square-shaped unstructured grid was constructed, ${ }^{32}$ and from this, a circular domain was created by removing all particles outside a circle centered at the domain center. The thick-walled cylinder was similarly created by also removing particles inside a smaller circle. The presented results are produced using a grid with 100.000 particles, while the grid refinement shown in Section 3.6 includes several grid resolutions from 1.000 to 250.000 particles. New models and meshing techniques aimed at further reducing the mesh bias will be considered for future studies, but is outside the scope of this work.

The mechanical equation system is set up by defining the force and torque acting on all particles. For a beam connecting particles $i, j$ (Figure 2), the force $\mathbf{F}_{i j}$ and torque $M_{i j}$ acting on particle $i$ are given by

$$
\begin{gathered}
\mathbf{F}_{i j}=k_{n}\left(L_{i j}-L_{i j, e q}\right) \mathbf{n}_{i j}+k_{\omega} L_{i j, e q}\left(\theta_{i j}+\theta_{j i}\right) \mathbf{s}_{i j}, \\
M_{i j}=2 k_{\omega} L_{i j, e q}^{2}\left(\gamma\left(\theta_{i j}-\theta_{j i}\right)+\frac{1}{3} \theta_{i j}+\frac{1}{6} \theta_{j i}\right),
\end{gathered}
$$

where $k_{n}$ is a spring constant, $L_{i j}$ is the distance between the particles, $L_{i j, e q}$ is the equilibrium distance, $\mathbf{n}_{i j}$ is a unit vector parallel to the beam, $k_{\omega}$ is a rotational spring constant, $\theta_{i j}$ and $\theta_{j i}$ are bending angles defined in Figure 2 , $\mathbf{s}_{i j}$ is a unit vector perpendicular to the beam, and $\gamma=\frac{1}{6} a^{2}\left(1+v_{0}\right)$. Here, $a=d / L_{i j, e q}$, where $d$ is the width of the beam. Furthermore, $k_{\omega}=k_{n}\left(2 / a^{2}+4\left(1+v_{0}\right)\right)^{-1}$. The input parameters $k_{n}, a$, and $v_{0}$ are adjusted to reproduce the wanted continuum scale elastic behavior. To make the model dimensionless, the parameters are adjusted to give a Young modulus equal to one. All stresses are then interpreted as fractions of the Young modulus of the modeled solid. The usage of a dimensionless formulation is a convenient choice, as this treatment makes it easier to compare numerical results against analytical solutions and to prevent the eigen-spectrum of the tangential matrix shifting due to the adoption of different unit systems.

A quasistatic approach is chosen for solving the equation system incrementally, in which it is assumed that the material immediately reaches an equilibrium configuration. This is achieved by finding the configuration of positions $x_{i}, y_{i}$ and rotation $\omega_{i}$, which gives a sum of forces equal zero for every particle $i$. For each beam, we use a linearized version of Equations 3 to 4 given by

$$
\begin{aligned}
& \left(k_{n} \cos ^{2} \theta+2 k_{\omega} \sin ^{2} \theta\right)\left(x_{i}-x_{j}\right)+\left(k_{n}-2 k_{\omega}\right) \sin \theta \cos \theta\left(y_{i}-y_{j}\right) \\
& -k_{\omega} L_{i j, e q} \sin \theta\left(\omega_{i}+\omega_{j}\right)+L_{i j, e q}\left(k_{n} \cos \theta+2 k_{\omega}\left(\theta-\theta_{\text {init }}\right) \sin \theta\right)=0
\end{aligned}
$$




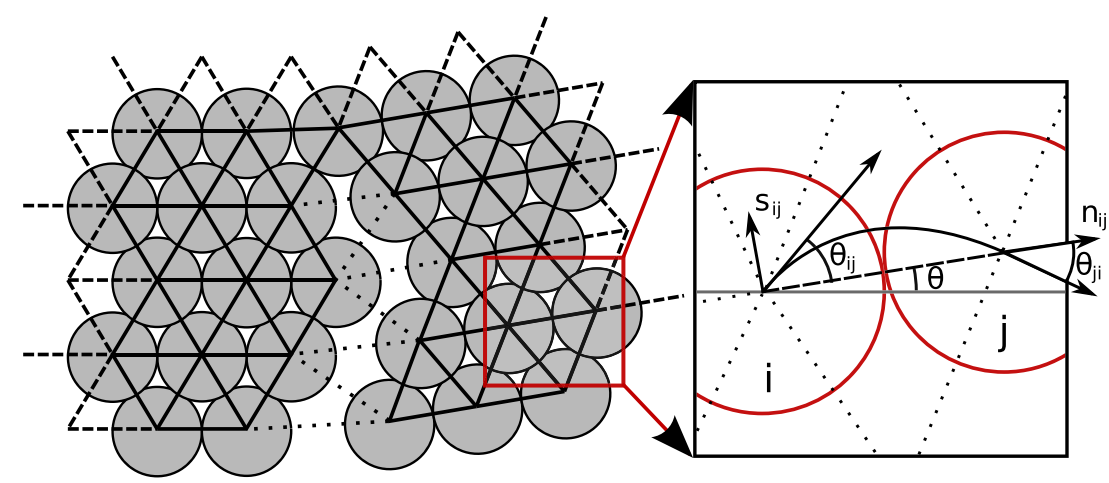

FIGURE 2 Left: A section of the prime grid used in the mechanical model, showing particles as circles and intact beams as solid lines. Dashed lines represent connections to surrounding particles (not shown), and the dotted lines represent beams that are removed due to fracture formation. In combination, intact and removed beams always form triangles in this model. We define such triangles consisting of 2 intact beams and 1 removed beam, as seen at the top of the central crack, to be a crack tip. The grid is shown as hexagonally close packed, for clarity. Right: Expanded view of the connection between particles $i, j . \theta$ is the angle between the horizontal and the direction of the unit vector $\mathbf{n}_{i j}$ pointing from particle $i$ to $j, \theta_{i j}$ and $\theta_{j i}$ represents the deflection of the beam at particles $i$ and $j$, respectively, (shown overexaggerated for clarity) and $\mathbf{s}_{i j}$ is a unit vector perpendicular to $\mathbf{n}_{i j}$ [Colour figure can be viewed at wileyonlinelibrary.com]

$$
\begin{aligned}
& \left(k_{n}-2 k_{\omega}\right) \sin \theta \cos \theta\left(x_{i}-x_{j}\right)+\left(k_{n} \sin ^{2} \theta+2 k_{\omega} \cos ^{2} \theta\right)\left(y_{i}-y_{j}\right) \\
& +k_{\omega} L_{i j, e q} \cos \theta\left(\omega_{i}+\omega_{j}\right)+L_{i j, e q}\left(k_{n} \sin \theta-2 k_{\omega}\left(\theta-\theta_{i n i t}\right) \cos \theta\right)=0 \\
& \quad-k_{\omega} L_{i j, e q} \sin \theta\left(x_{i}-x_{j}\right)+k_{\omega} L_{i j, e q} \cos \theta\left(y_{i}-y_{j}\right) \\
& +2 k_{\omega} L_{i j, e q}\left(\left(\gamma+\frac{1}{3}\right) \omega_{i}-\left(\gamma-\frac{1}{6}\right) \omega_{j}\right)-k_{\omega} L_{i j, e q}^{2}\left(\theta-\theta_{i n i t}\right)=0,
\end{aligned}
$$

where $\theta_{\text {init }}$ is the angle of the beam in the initial configuration of the grid. A brittle fracture is represented by the irreversible removal of a beam when the stress becomes too high locally. After removal of a beam, there will be no tensile force exerted on the contact pair and the compressive forces are exerted only if 2 particles are overlapping and therefore lead to closure of cracks. This path-dependent behavior is enforced by labeling the contact pair in a damaged state, a technique commonly used in element-deletion methods in finite element fracture models. ${ }^{51,52}$ Thus, forces are in this case given by

$$
\mathbf{F}_{i j}=k_{n}\left(L_{i j}-L_{i j, e q}\right) H\left(L_{i j, e q}-L_{i j}\right) \mathbf{n}_{i j}
$$

$H$ is the Heaviside step function, which sets the force to zero when the particles are nonoverlapping. In linearized form for overlapping particles, this becomes

$$
\begin{aligned}
& k_{n} \cos ^{2} \theta\left(x_{i}-x_{j}\right)+k_{n} \sin \theta \cos \theta\left(y_{i}-y_{j}\right)+k_{n} L_{i j, e q} \cos \theta=0, \\
& k_{n} \sin \theta \cos \theta\left(x_{i}-x_{j}\right)+k_{n} \sin ^{2} \theta\left(y_{i}-y_{j}\right)+k_{n} L_{i j, \text { eq }} \sin \theta=0 .
\end{aligned}
$$

Fracture formation is based on the local stress tensor $\sigma_{i}$, which is computed for each particle $i$ using the sum of outer products $\sigma_{i}=0.5 \cdot V_{i}^{-1} \Sigma_{j}\left(\mathbf{F}_{i j} \otimes \mathbf{L}_{i j}\right),{ }^{53}$ where the sum over $j$ represents all particles connected to or in contact with particle $i, V_{i}$ is the volume of particle $i, \mathbf{F}_{i j}$ is the force between particles $i, j$ found using Equation 3 or 8 , and $\mathbf{L}_{i j}$ is the distance vector from particle $i$ to particle $j$. Stresses computed using this method have been benchmarked against continuum-based methods. ${ }^{32}$ The stress state in each beam is assumed to be equal to the mean value of the stress in the particles that it connects. We model failure using the Coulomb criterion, ${ }^{54}$ rewritten in the form:

$$
\sigma_{C C}=\left[\left(1+\mu_{i}^{2}\right)^{1 / 2}+\mu_{i}\right]^{2} \sigma_{1}-\sigma_{3}>C_{u},
$$

where $\sigma_{1}$ and $\sigma_{3}$ represent the major and minor principal stress, respectively, $\mu_{i}$ is the internal friction coefficient, and $C_{u}$ is the uniaxial compressive strength of the material, defining the situation where a shear fracture will occur. We will in 


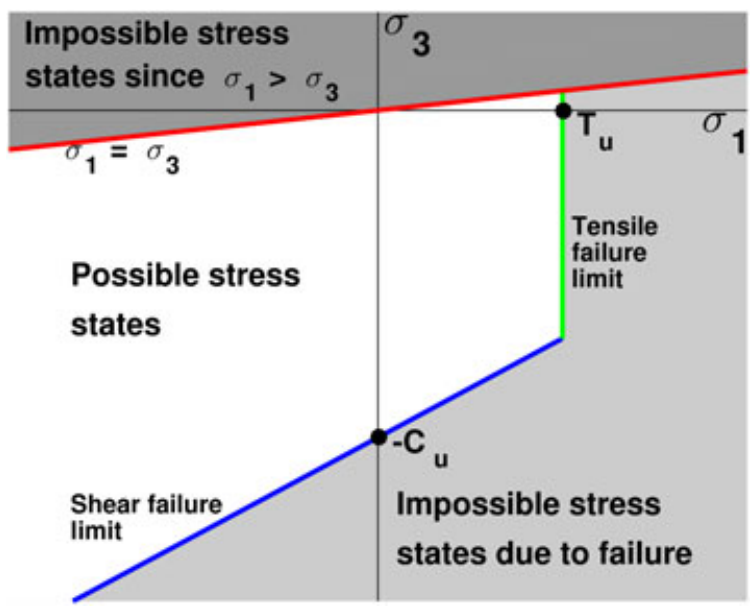

FIGURE 3 Illustration of the fracture criteria used in this work. The stress states above the line $\sigma_{1}=\sigma_{3}$ are impossible states, because per definition $\sigma_{1}>\sigma_{3}$. Above the tensile failure limit $\sigma_{1}=T_{u}$, a tensile fracture is formed, and below the shear failure limit defined by Equation 11, the material fails in shear. Stress states outside the failure limits are impossible due to fracture formation. Notice that the scale of the $\sigma_{1}$ and $\sigma_{3}$ axes are different to enhance visibility [Colour figure can be viewed at wileyonlinelibrary.com]

the following use the term coulomb stress to refer to $\sigma_{C C}$. In addition, we have the tensile criterion $\sigma_{1}>T_{u}$, in which $T_{u}$ is the uniaxial tensile strength of the material, giving the state at which a tensile fracture will form. As seen in Figure 3 , depending on the situation, either of the 2 criteria can be fulfilled first.

Near a fracture tip, the stress in a material decays with the square root of distance from the tip..$^{55}$ In our mechanical model, we define a fracture tip to be present in any triangle formed from 2 intact beams and 1 broken beam (see Figure 2). Thus, when the stress in the center of an intact beam with length $L_{i j}$ present in such a triangle is computed by averaging the stress in particles $i, j$, the stress is underestimated by a factor proportional to $L_{i j}^{1 / 2}$. This causes a grid resolution dependence, since low resolution necessarily leads to longer $L_{i j}$ than high resolution, and thus, fractures will tend to grow faster on high resolution grids than in low resolution grids. To correct for this difference, we multiply the stress in a beam with length $L_{i j}$ at a crack tip with a factor $\left(L_{i j} / 2 L_{m}\right)^{1 / 2}$, where $L_{m}$ is a material parameter describing the typical radius of curvature of a crack tip.

Two different mechanical boundary conditions are used in this work, either a fixed boundary or a free boundary. By assuming that the domain is located in an isotropic external stress field of magnitude $b p_{0}$, where $p_{0}$ is the initial fluid pressure in the domain, the mechanical stress is zero everywhere in the initial state, and the boundary is free to deform. Boundaries are in this DEM formulation mechanically free unless confinement is included. A fixed boundary is implemented by setting constant $x$ - and $y$-coordinates for particles at the fixed boundary.

When assembling contributions from all beams and contacts according to Equations 5 to 7 and 9 to 10 with relevant boundary conditions, the result is a linear system of equations for positions $x_{i}, y_{i}$ and rotation $\omega_{i}$ for all particles $i$. Additional terms related to fluid pressure applied to the particles are added to this equation system in Section 2.3, before the solution strategy using a conjugate gradient method is presented.

\section{2 | Fluid transport model}

Fluid transport is modeled using Equation 2 with a source term, which is rewritten using an integral over volume and Gauss' theorem to obtain

$$
\frac{1}{M} \int_{V} \frac{\partial p}{\partial t} \mathrm{~d} V-\int_{S} \frac{k}{\mu} \nabla p \cdot \mathbf{n} \mathrm{d} S+b \int_{V} \frac{\partial \varepsilon_{v}}{\partial t} \mathrm{~d} V=\int_{V} q \delta(r) \mathrm{d} V
$$

where $V$ is the volume over which the equation is integrated, $S$ denotes the surface of the volume $V$, and $\mathbf{n}$ is an outwards facing unit vector from the surface $S . M$ denotes Biot modulus, and $k / \mu$ is the hydraulic conductivity. ${ }^{56}$ The right hand side term is a source term for a point injection of magnitude $q$ at the center of the domain, and $\delta(r)$ is a delta function for radial position. From here on, all volumes and areas are given per unit length in the axial direction, so that the unit of a volume is $\left[\mathrm{m}^{3}\right] /[\mathrm{m}]=\left[\mathrm{m}^{2}\right]$, and the unit of an area is similarly $[\mathrm{m}]$. We now introduce a dimensionless formulation by 
defining the dimensionless variables $p^{*}=\frac{p-p_{0}}{E}, V^{*}=\frac{V}{R^{2}}, S^{*}=\frac{S}{R}, \nabla^{*}=R \nabla$, and $t^{*}=\frac{t}{t_{\mathrm{s}}}$, where $R$ is the domain radius, $E$ is Young modulus, $p_{0}$ is the constant initial pressure level, and $t_{s}$ is a time scale yet to be decided. With these definitions and a slight rearrangement, Equation 12 becomes

$$
\frac{E}{M} \int_{V^{*}} \frac{\partial p^{*}}{\partial t^{*}} \mathrm{~d} V^{*}-\int_{S^{*}} \frac{k E t_{s}}{\mu R^{2}} \nabla^{*} p^{*} \cdot \mathbf{n} \mathrm{d} S^{*}+b \int_{V^{*}} \frac{\partial \varepsilon_{v}}{\partial t^{*}} \mathrm{~d} V^{*}=\frac{t_{s}}{R^{2}} \int_{V^{*}} q \delta(r) \mathrm{d} V^{*}
$$

When choosing time scale $t_{s}=\frac{\mu R^{2}}{k E}$ and introducing a dimensionless point injection rate $Q=\frac{\mu q}{k E}$, the equation simplifies further to

$$
\frac{E}{M} \int_{V^{*}} \frac{\partial p^{*}}{\partial t^{*}} \mathrm{~d} V^{*}-\int_{S^{*}} \nabla^{*} p^{*} \cdot \mathbf{n} \mathrm{d} S^{*}+b \int_{V^{*}} \frac{\partial \varepsilon_{v}}{\partial t^{*}} \mathrm{~d} V^{*}=\int_{V^{*}} Q \delta(r) \mathrm{d} V^{*}
$$

To discretize this expression, we now introduce a dual grid. When particles are closely packed, the combination of intact and removed beams in the DEM can be regarded as the edges in a Delaunay triangulation. From here on, we denote this triangulation the prime grid. A Voronoi tesselation is constructed by drawing equidistant lines between particles that are nearest neighbors, perpendicular to the connection between the particles. In a valid Delaunay triangulation, each triangle edge will correspond to one line segment in the Voronoi tessellation (Figure 4), and each Delaunay triangle will correspond to one connection point between 3 segments in the Voronoi tessellation. The grid constructed this way is denoted the dual grid. We choose to solve for the evolution of pressure $p_{s}$ at the Voronoi connection point of each Delaunay triangle $s$ and let the integration volume $V^{*}$ be the volume of the Delaunay triangle, thus making the surface $S^{*}$ of the volume the triangle's 3 facets. Fluid is then allowed to flow along each Voronoi segment connecting triangles $s, t$. To keep the notation clean, we from here on drop the asterisk $\left(^{*}\right)$ on scaled parameters. Discretized for time steps $n$ using the dual grid for triangles where $\delta(r)=0$, Equation 14 becomes

$$
\frac{V_{s}^{n+1}}{M}\left(p_{s}^{n+1}-p_{s}^{n}\right)-\sum_{t} \frac{\Delta t A_{s t}^{n+1}}{d_{s t}^{n+1}}\left(p_{t}^{n+1}-p_{s}^{n+1}\right)+b\left(V_{s}^{n+1}-V_{s}^{n}\right)=0
$$

where $\Delta t$ is the time step, $A_{s t}$ denotes the length of the facet between triangles $s$ and $t, d_{s t}$ is the length of the Voronoi segment, $V_{s}$ denotes the volume of triangle $s$, and the sum runs over all Voronoi segments that are connected at triangle $s$. For a triangle that contains the point $r=0$, the right hand side becomes $Q \Delta t$ to include the source term. For Voronoi segments that terminate at a domain surface (Figure 4 ), $d_{s t}$ is replaced by the distance from the Voronoi connection point of triangle $s$ to the surface $\left(d_{s u}\right)$, and $p_{t}$ is replaced by the fluid pressure at that surface. The pressure is assumed constant at the outer boundary, while the borehole pressure develops in time according to

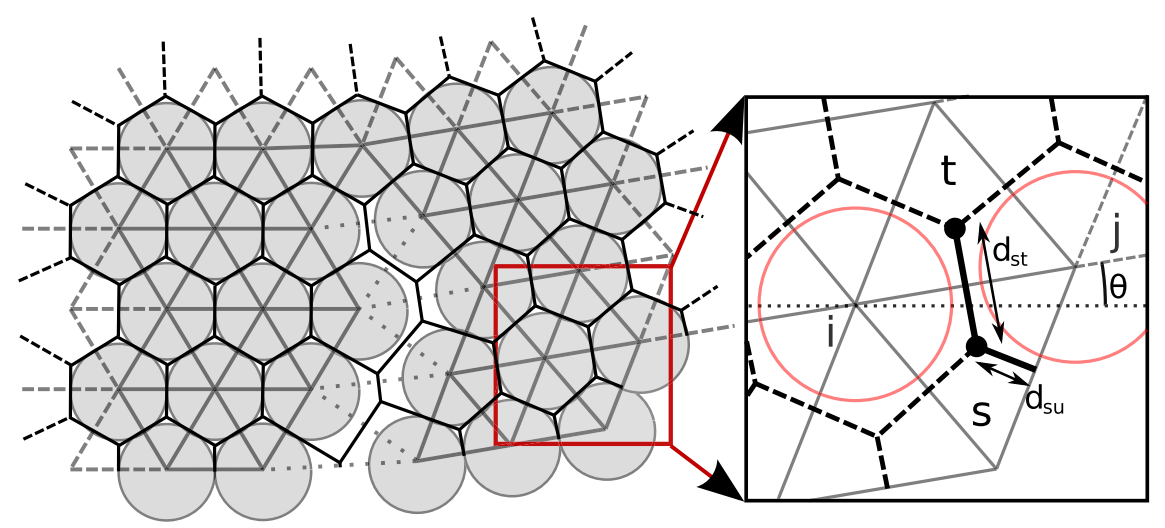

FIGURE 4 Definition of the dual grid used in the fluid transport model. The grid is shown as hexagonally close packed, for clarity. Left: The prime grid introduced in Figure 2 forms a Delaunay triangulation of the domain, shown as gray solid, dashed, and dotted lines. The black lines form a Voronoi tessellation, which is the dual grid. Dashed black lines represent connections to surrounding material. Right: Expanded view of the grid. By definition, the Voronoi segment of length $d_{s t}$ connecting triangles $s$ and $t$ is perpendicular to the edge between the triangles. For an intact beam between particles $i$ and $j, \theta$ is the angle of the beam. When a Voronoi segment reaches the surface, it is terminated at the facet of the triangle, as seen in triangle $s$ [Colour figure can be viewed at wileyonlinelibrary.com] 


$$
\frac{V_{h}^{n+1}}{M}\left(p_{h}^{n+1}-p_{h}^{n}\right)-\sum_{t} \frac{\Delta t A_{h t}^{n+1}}{d_{h t}^{n+1}}\left(p_{t}^{n+1}-p_{h}^{n+1}\right)=-b\left(V_{h}^{n+1}-V_{h}^{n}\right)+Q \Delta t,
$$

where $h$ denotes the borehole and the sum runs over all triangles $t$ along the perimeter of the borehole.

Fractures can be introduced in this model by increasing $k$ for Voronoi segments that correspond to broken beams by a factor $\imath$. In this work, we let $\iota$ be a constant and ignore the effect of the fracture aperture. When contributions from all connections between triangles and between triangles and surfaces are assembled, it gives a linear system of equations for pressure $p_{s}$, which is solved using a conjugate gradient method.

\section{3 | Two-way coupling of mechanical and fluid models}

Models that capture multiphysical coupling processes often lead to a system of equations with multiple sets of variables (eg, displacement, chemical concentration, pore pressure, temperature, and Lagrangian multipliers). The most direct approach is to solve the resulting system of equations via a monolithic multiphysics solver. ${ }^{57-62}$ In this case, the governing equations that represent the multiphysical coupling processes are treated as one entity such that all unknown variables advance or march together in time.

Another possibility is to solve the multiple governing equations via sequential iterations between solid and fluid solvers. This idea can be traced back to the operator-splitting concept and has been widely used in continuum poromechanics problems. ${ }^{63-66}$ The key of the splitting approach is to "freeze" or make an approximated update on a subset of unknown variables and thereby temporarily decouple a large system of equations into 2 or more subsystems. This predictor step is then followed by one or multiple correction steps that update(s) the rest of the unknowns and enforce the consistency of all constraints. If solved properly, solutions sought via the monolithic and sequential iterative coupling solvers should yield identical results.

In this study, a family of sequential coupling schemes is used to capture the hydromechanical coupling between the solid skeleton and the pore-fluid. Based on previous works, ${ }^{63,64}$ the drained and undrained operator split will be adopted to establish the hydromechanical coupling between the DEM and flow network solvers. Both the drained and undrained split algorithms involves a predictor step that updates the solid displacement via the balance of linear momentum. In the drained-split hydromechanical scheme, the mechanical step is updated while assuming that the fluid is close to a drained condition. Therefore, the fluid pressure is frozen at the pressure in the previous time step in the solid iterative step and later corrected by the fluid solver. The undrained split scheme approximates an updated pore pressure based on the assumption that pore-fluid is trapped inside the control volume in the mechanical step. In both cases, the mechanical step is followed by a fluid step in which the new pore pressure is found using Equation 15. The fluid step is identical for the drained and undrained split and is not discussed further.

In both drained and undrained splits, iterations between the fluid and solid solvers are often required to reach the equilibrium of the two-coupled hydromechanical system. Previous works on the sequential solver for continuum models indicate that the difference between the drained and undrained split is not significant when the permeability is high, but the drained split may require an order or more additional iterations when the permeability is low due to the lack of direct feedback from the solid solver step. ${ }^{66}$ However, other works ${ }^{63,65}$ have shown that the drained split strategy may lead to nonconvergent results even for linear poroelasticity problems. In this work, our primary focus is on the undrained split. However, for comparison, the drained split is also included.

To prescribe traction exerted on the surface of a particle by the pore fluid constituent, the Voronoi segments surrounding a particle are chosen as the surface the fluid pressure acts on. This treatment ensures that the pressure acts on a surface that encloses the entire particle: A particle in a constant pressure field will feel zero net force, as the traction exerted on the solid surface by the pore fluid is in balance. We assume that in a Voronoi segment that connects Delaunay triangles $s, t$, the pressure acting on the 2 nearby particles $i, j$ (see Figure 4$)$ is $p_{s t}=0.5\left(p_{s}+p_{t}\right)$, where $p_{s}$ and $p_{t}$ again denote the pressure in triangles $s, t$.

The force acting on particle $i$ (Figure 4) due to the fluid pressure in Voronoi segment $s, t$ is now given by

$$
\mathbf{F}_{s t, i}=-\xi b d_{s t} p_{s t}\left(\begin{array}{c}
\cos \theta \\
\sin \theta
\end{array}\right)
$$


where $\xi$ is an empirical scaling constant adjusted to fit continuum scale behavior. Notice that the fluid and solid models use the same dimensionless formulation: Both stresses and pressures are normalized by Young modulus, thus making the 2 dimensionless models compatible.

In the undrained split scheme, one makes the assumption that the pore fluid is temporarily in an undrained condition, and the void space is assumed to hold the same amount of fluid during the solid step. As a result, the volume change of the solid skeleton will change the void space size during a time step from time $n$ to $n+1$ and cause a pressure change according to

$$
p_{s t, e f f}=p_{s t}-\frac{b M}{2 E}\left(\frac{V_{s}^{n+1}-V_{s}^{n}}{V_{s}^{n}}+\frac{V_{t}^{n+1}-V_{t}^{n}}{V_{t}^{n}}\right) .
$$

The pore pressure in (18) can be viewed as a predictor pore pressure that is later corrected by the fluid solver. To incorporate the predictor pore pressure into the discrete element solver, the volume terms in (18) must be written in terms of the degrees of freedom of the solid constituent, ie, the particle displacement. In the grain contact network, 3 contacting particles may form a so-called three-cycle. ${ }^{67}$ When 2 three-cycles share an edge, together they make up a convex quadrilateral, for which the area is given by $A_{\text {quad }}=\frac{1}{2} b c \sin \zeta$, where $b, c$ are the lengths of the diagonals of the quadrilateral and $\zeta$ is the angle between the diagonals. One of the diagonals is the distance between particles $i, j$. It is now assumed that the other diagonal and the angle between the diagonals are unchanged during the mechanical relaxation, which leads to the linearized expression

$$
p_{s t, e f f}=p_{s t}-\frac{b M}{E} \frac{L_{i j}^{n+1}-L_{i j}^{n}}{L_{i j}^{n}},
$$

where $L_{i j}$ denotes the distance between the centers of particles $i, j$. This leads to a linearized force on particle $i$ given by

$$
\mathbf{F}_{s t, i}=-\xi b d_{s t}\left[p_{s t}+\Psi\left(\frac{b M}{E}-\frac{b M}{E L_{i j}^{n}}\left(\left(x_{j}-x_{i}\right) \cos \theta+\left(y_{j}-y_{i}\right) \sin \theta\right)\right)\right]\left(\begin{array}{c}
\cos \theta \\
\sin \theta
\end{array}\right),
$$

where $\Psi$ is a parameter that changes the extent of feedback from the mechanical deformation and can be adjusted to achieve optimal convergence of iterations in the operator split. Notice that the endmember $\Psi=0$ is the drained split, with no feedback. Clearly, a too high $\Psi$ will cause too much feedback, and an optimal choice for $\Psi$ will reduce the number of iterations necessary. When adding pressure contributions for all Voronoi segments to the linear system of equations for mechanical deformation given by Equations 5 to 7 and 9 to 10, this defines the effect of fluid pressure on the particles.

The solution strategy for the final equation system describing mechanical deformation and pressure evolution is presented in Figure 5. At the start of each time step, the mechanical system (Equations 5-7, 9-10, and 20) is set up with $\Psi=0$ for a drained system and nonzero $\Psi$ for an undrained system. The mechanical system is then solved using a conjugate gradient method, thus finding a new equilibrium configuration of particles, before the properties of the flow network are recomputed for this time step. If fracture formation is included in the present simulation, the stress in the current configuration is computed, and if any beams exceed the fracture criteria, the beam with the highest stress is removed. If a beam is removed, the system is set up and solved again as outlined above, before recomputing the stress, until no beams exceed the fracture criteria. This is the same approach as used previously for a purely mechanical DEM, without the fluid coupling. ${ }^{32,33}$ Then the fluid transport system (Equations 15-16) is set up based on the current configuration of the flow network and solved using a conjugate gradient method. If the operator split is iterated, the entire process is then started over again. Iterations continue until the current pressure and particle positions simultaneously represent an equilibrium state of the mechanical system (Equations 5-7, 9-10, and 20) and a solution of the fluid transport system (Equations 15-16). Then the solver proceeds to the next time step.

\section{4 | Model assumptions, parameter estimates, and scaling behavior}

We assume that the material studied in this work is initially situated in an isotropic compressive stress field that exactly matches the stress contribution from an initial fluid pressure $p_{0}$ so that Equation 1 becomes

$$
\boldsymbol{\sigma}=\boldsymbol{\sigma}_{m}+b \boldsymbol{\sigma}_{f}=-b p_{0} \boldsymbol{I},
$$




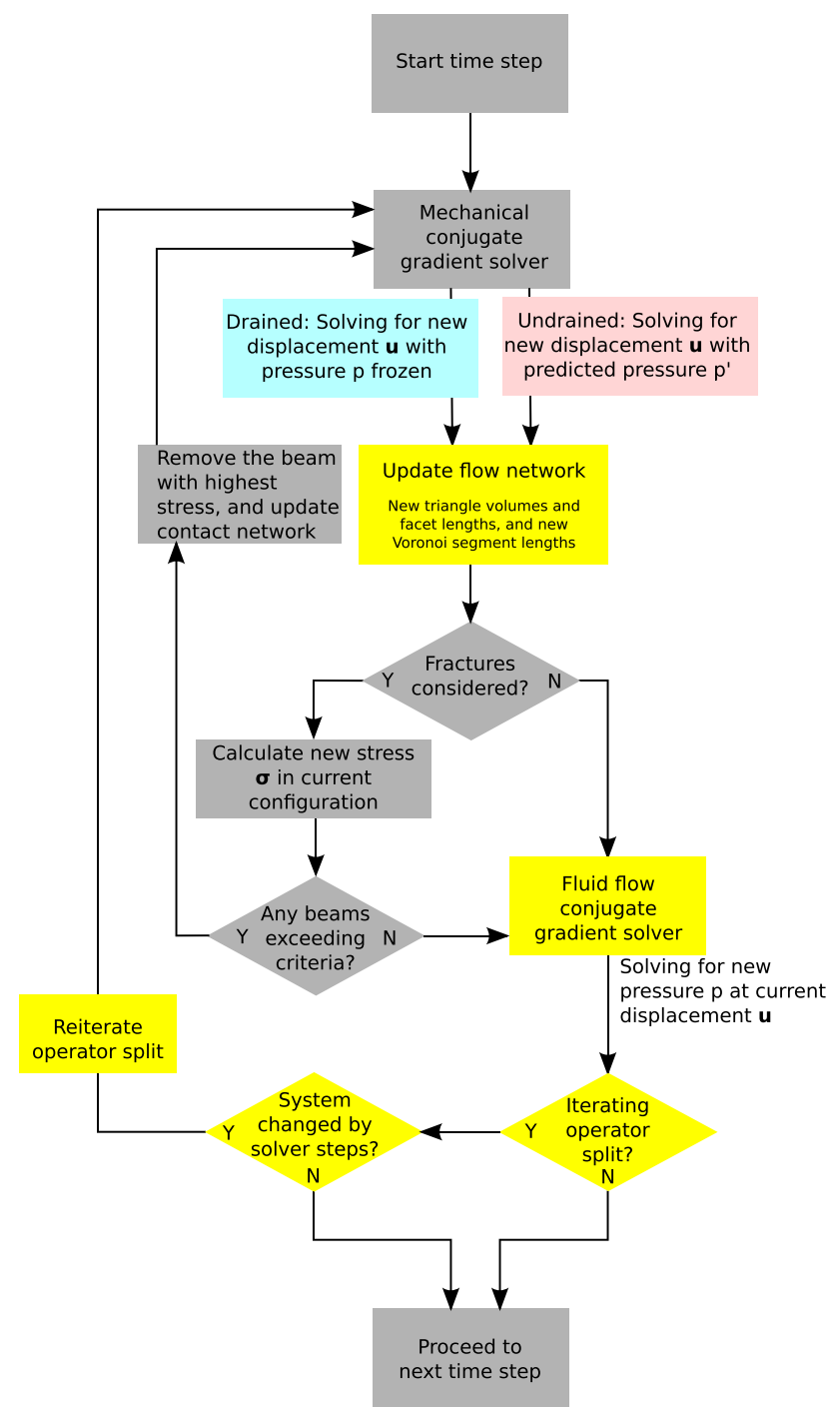

FIGURE 5 Flow chart of the undrained and drained-split scheme for the discrete hydromechanical model. If the operator split is iterated and the system is changed during the solver steps, a new iteration is necessary to ensure that both solvers are converged in the current system state. The gray boxes are necessary parts of a mechanical discrete element model, ${ }^{32,33}$ while the yellow boxes are the additional pieces necessary for the fluid coupling. The choice of drained or undrained split is highlighted by different colors [Colour figure can be viewed at wileyonlinelibrary.com]

where $\boldsymbol{I}$ denotes the identity matrix. Thus, the material is initially stress free; $\sigma_{m}=0$. From here on, we neglect the compressive stress field and the initial fluid pressure, since they cancel. Thus, we let the material be stress free at the boundary and discuss pressure $p$ as deviations from $p_{0}$. This allows negative pressures in the model, which we interpret as a fluid pressure lower than $p_{0}$. We further assume that the material remains fluid saturated at any negative pressure, which means that we assume that the absolute pressure remains above the vapor pressure of the fluid.

The assumed parameters and parameter ranges are shown in Table 1. Based on these parameters, the Biot modulus $M$ can be found to be

$$
M=\frac{1}{\frac{\Phi}{K_{w}}+\frac{3(b-\Phi)(1-\Phi)(1-2 v)}{E}} .
$$

From the dimensionless formulation derived in Section 2.2, it is obvious that the relevant time scales for a fluid injection process are $t_{s}=\frac{\mu R^{2}}{k E}$, which is related to the Darcy velocity of the fluid flow, and $t_{q}=\frac{R^{2}}{q}$, which is a time scale related to the fluid injection rate. Notice that since the mechanical model is quasistatic, it has no inherent time scale. Thus, $t_{s}$ and $t_{q}$ are the only 2 time scales. The ratio of these, $Q=\frac{\mu q}{k E}$, is a dimensionless parameter. A low $Q$ means that a significant fraction of the injected fluid is able to leave the domain through porous flow, in what Detournay ${ }^{68}$ denoted the leak-off 
TABLE 1 Assumed parameter ranges

\begin{tabular}{llll} 
Parameter & Symbol & Range & Unit \\
\hline Young modulus & $E$ & $10^{10}$ & $\mathrm{~Pa}$ \\
Poisson ratio & $v$ & 0.25 & - \\
\hline Uniaxial tensile strength & $T_{u}$ & $2 \cdot 10^{6}$ & $\mathrm{~Pa}$ \\
\hline Uniaxial compressive strength & $C_{u}$ & $3.33 \cdot 10^{7}$ & $\mathrm{~Pa}$ \\
\hline Internal friction coefficient & $\mu_{i}$ & 0.9 & - \\
\hline Permeability & $k$ & $10^{-17}-10^{-14}$ & $\mathrm{~m}^{2}$ \\
\hline Permeability in fractures & $k_{f}$ & $10^{-14}-10^{-11}$ & $\mathrm{~m}^{2}$ \\
\hline Viscosity & $\mu$ & $10^{-3}$ & $\mathrm{~Pa} \cdot \mathrm{s}$ \\
\hline Biot coefficient & $b$ & $0.2-0.8$ & - \\
\hline Porosity & $\Phi$ & $10^{-3}$ & - \\
\hline Fluid bulk modulus & $K_{w}$ & $2.2 \cdot 10^{9}$ & $\mathrm{~Pa}$ \\
\hline Injection rate per unit axial length & $q$ & $10^{-3}-10^{-1}$ & $\mathrm{~m}^{2} / \mathrm{s}$ \\
\hline Domain outer radius & $R$ & 1 & $\mathrm{~m}$ \\
\hline Cylinder inner radius & $R_{i}$ & 0.138 & $\mathrm{~m}$ \\
\hline
\end{tabular}

regime. On the other hand, high $Q$ can cause a significant pressure buildup, which is closer to the impermeable regime. In the following, we will focus on the effect of changing the injection rate $Q$, the permeability in fractures $k_{f}$, and the Biot coefficient $b$. All dimensionless times are reported using $t_{s}$ as time scale.

The final relevant dimensionless parameters for the fluid transport are the ratio between Young modulus $E$ and the Biot modulus $M$, the ratio between the outer and inner radii of the domain $\frac{R_{i}}{R}$, which is zero for a point injection into a solid, and the ratio between the permeability in fractures and bulk rock permeability, $l=k_{f} / k$. For a borehole, we choose the same radii ratio as used by Grassl et al. ${ }^{26}$ Notice that even though $\Phi$ is a dimensionless parameter, it enters Equation 12 through $M$ only and can be conveniently included in the dimensionless parameter $\frac{E}{M}$.

The dimensionless parameters controlling the mechanical system are Poisson ratio $v$, tensile strength $\frac{T_{u}}{E}$, compressive strength $\frac{C_{u}}{E}$, and internal friction coefficient $\mu_{i}$, in addition to the Biot coefficient $b$. We do not intend to study the effect of changing purely mechanical parameters in any detail and focus on the settings summarized in Table 1.

It is immediately clear that in the present formulation, the time scales $t_{s}, t_{q}$ can be changed freely while keeping $Q$ and $E$ constant, without changing Equation 14 . Thus, rapid injection into a high-permeability rock will proceed exactly as a slow injection into a suitably tight rock, but at a shorter time scale. Similarly, changing system size $R$ changes the time scale only, in a relation characteristic for a pressure diffusion process. This scaling behavior is a result of the lack of time scale in the mechanical model. A more advanced mechanical model might include time-dependent processes, eg, viscous relaxation, but this is determined to be outside the scope of the present work.

\section{3 | RESULTS}

In this section, we first present as a benchmark problem point injection into a finite domain in 2D DEM and compare the convergence rate for the drained and undrained operator splits. Then we turn our attention to continuous fluid injection into a borehole. Firstly, a basic understanding of the system is achieved by studying the pressure development and deformation in a simplified 1D geometry, disregarding any fracture formation. Then we study the same systems in 2D DEM, including fracture formation and the importance of the coupling with faster fluid transport in fractures. Finally, we focus on the borehole breakdown pressure, before we present results showing the effect of changing grid resolution.

\section{1 | Benchmark: pressurized cylindrical cavity}

The injection of fluid into porous media is a problem of great interest in environmental engineering, biomechanics, and petroleum engineering. Not surprisingly, this interest has led to the development of analytical solutions for several idealized situations. One class of settings that has been extensively studied is the pressurization of a preexisting fracture in an impermeable rock. Another class involves coupled fluid flow in and deformation of a continuous porous material. One of the most commonly used analytical solutions in this class is derived for the pressured cylindrical cavity problem in the plane strain setting. ${ }^{1,2,56,69}$ Since the pressurized fracture problem only involves the effect of fluid pressure on the 


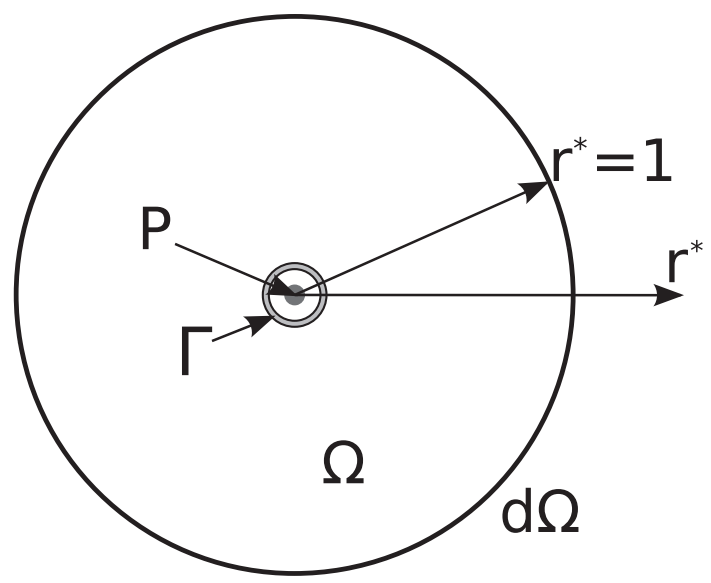

FIGURE 6 Geometry for the point injection setting. Fluid is continuously injected in the center of domain $\Omega$ at point $P$ and flows in the $r^{*}$-direction. The boundary $d \Omega$ at $r^{*}=1$ is either mechanically free or fixed. The cylindrical shell $\Gamma, 0.02<r^{*}<0.03$ (size overexaggerated for clarity), defines triangles for which pressure convergence is studied in Figure 8

solid, it does not require the full two-way coupled model. Thus, we have included an example of a pressurized fracture in Appendix C, and here, focus on the full two-way coupled model applied to the cylindrical cavity problem.

The pressured cylindrical cavity problem can be described as follows. Consider a homogeneous elastic half-space subjected to a sudden continuous mass source of pore fluid. The fluid infiltrated porous elastic solid near the fluid mass source will first deform in an undrained manner when load alternations are too rapid to allow pore fluid to diffuse. Over time, the diffusion of pore fluid leads to deformation further in the radial direction. Eventually, given sufficient time, the poroelastic responses will transit from the undrained limit to the drained limit where there is no alternation in pore pressure. ${ }^{2}$ In this numerical example, the pressured cylindrical cavity is re-introduced as a benchmark problem to verify the implementation of the two-way coupling DEM-network model. As pointed out, ${ }^{70,71}$ this verification exercise is essential to ensure that the numerical model accurately represents and correlates to the governing equations (poroelasticity) intended to be solved and hence establish credibility to the modeling effort.

Since it is not trivial to introduce an elastic half space in the DEM-network model, a circular domain is used (see Figure 6). As a result, the comparisons between the analytical and numerical simulations will focus on the early and transition regimes where the pressure plume developed during the injection has not yet reached the outer boundary of the simulation domain to minimize the boundary effect.

The results of point injection simulations are shown in Figure 7, where the settings $Q=0.1$ and $b=0.5$ have been used. One may notice that the results generally fit the analytical solution ${ }^{2}$ well. Since the mechanical boundary conditions in the simulations and in the analytical solution are not identical, there is a displacement deviation far from the injection point, where a free boundary leads to a larger displacement and a fixed boundary naturally leads to a displacement that goes to zero at the domain perimeter. Further, it is evident that when using the undrained split, there are no significant differences in the results when running iterations until both the mechanical equation system and the fluid transport model are in equilibrium at the same time and using just one iteration of both systems. The drained split also leads to an almost identical result.

Figure 8 shows the pressure evolution during iterations in the operator split. It is clear that the drained split $(\Psi=0)$ leads to oscillations, as previously seen in other works. ${ }^{66}$ On the other hand, too high $\Psi$ causes slow convergence without any oscillations. Although $\Psi=1.3$ is seemingly close to the optimal choice, the total computational time until reaching the state shown in Figure 7 does not show significant variations for $1 \leq \Psi \leq 2$. However, the computational time is approximately doubled by using $\Psi=10$ and increases by a factor about 5 when using the drained split.

\subsection{Insights from $1 D$}

The computational efficiency of the 1D model presented in Appendix A facilitates a large number of simulations that elucidate system behavior, and the main points are presented here. The geometry is a radial section along $r^{*}$ of a circular domain with a borehole (see Figure 9), and the initial condition is a water-saturated material with mechanically free boundaries, zero fluid pressure at the outer boundary, and a continuous fluid injection into the borehole. 

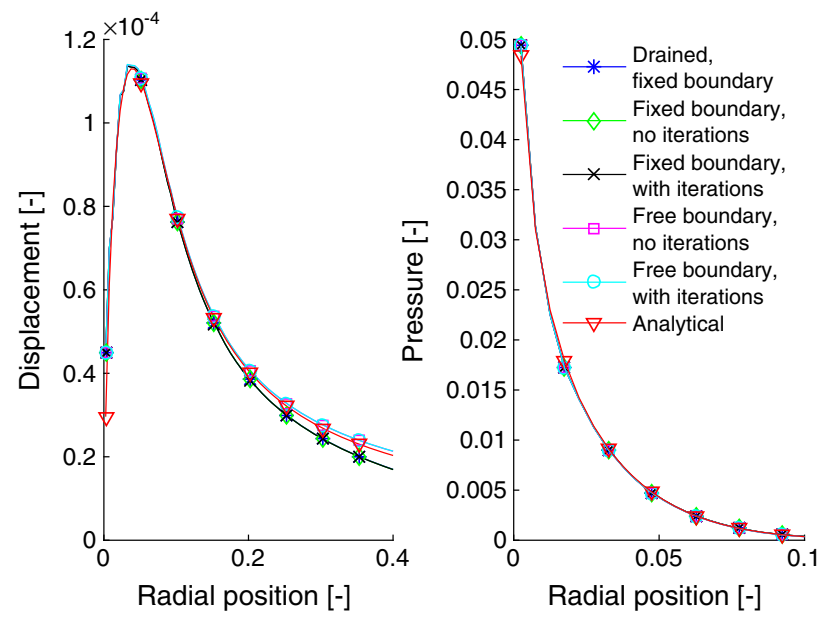

FIGURE 7 Results of point injection simulations at time $t^{*}=3 \cdot 10^{-4}$ with either a mechanically free surface or a fixed surface at radius $r^{*}=1$, with analytical solution from Rudnicki. ${ }^{2}$ The legend is for both subfigures. Left: Radial displacement $u_{r}^{*}$ near the domain center. All solutions are nearly identical to the analytical solution near the injection point, but a different mechanical boundary condition leads to a deviation further away from the domain center. With a fixed boundary, the displacement is underestimated, and with a free boundary, the displacement is overestimated. Different results with identical boundary conditions are nearly indistinguishable. Right: Pressure distribution $p^{*}$ near the domain center. All results are close to the analytical solution. Further away from the injection point, the pressure is close to zero. Both displacement and pressure values are mean values calculated at each radial position [Colour figure can be viewed at wileyonlinelibrary.com]

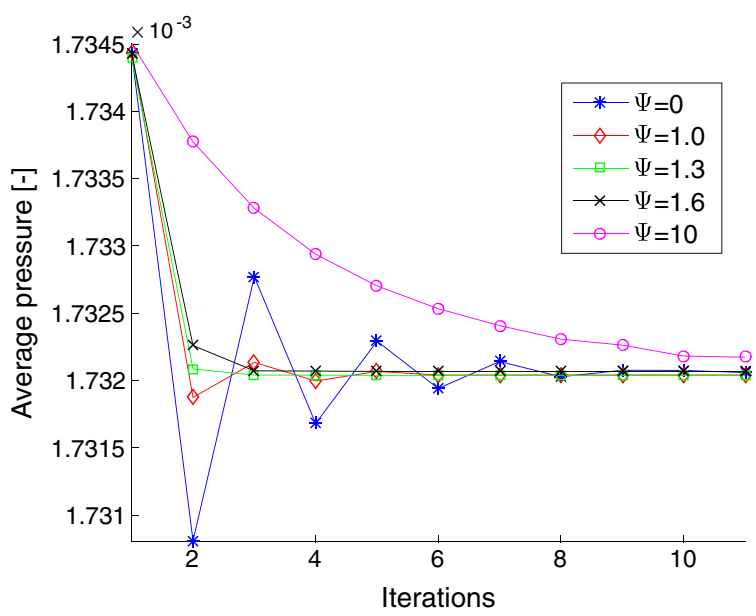

FIGURE 8 Pressure convergence within the cylindrical shell defined in Figure 6 for different values of $\Psi$. For $\Psi=0$, the pressure oscillates several times, while $\Psi=10$ causes slow convergence without iterations. The results suggest that $\Psi=1.3$ is close to the optimal choice for $\Psi$ in this case [Colour figure can be viewed at wileyonlinelibrary.com]

A typical example of results in time is shown in Figure 10. Obviously, the pressure increases in time (Figure 10A), first near the borehole, and later also closer to the rim. The final pressure fits almost precisely the analytical solution from Appendix B. As initially shown by Grassl et al, ${ }^{26}$ the fluid pressure in the borehole at steady state is given by

$$
p_{h}^{*}=-\frac{Q}{2 \pi} \ln R_{i}^{*},
$$

which shows the expected behavior: The borehole pressure at steady state increases with $Q$ and is higher for small boreholes. The analysis in Appendix B thus shows that with increasing $Q$, both displacement and the magnitude of all effective stress components at steady state increase. Furthermore, the steady state pressure does not depend on $b$, which means that the coupling between the mechanical model and the fluid flow model does not affect the steady state. The time development of pressure is only weakly dependent on $b$, with a borehole pressure increasing faster for $b=0.0$ and reaching $99 \%$ 


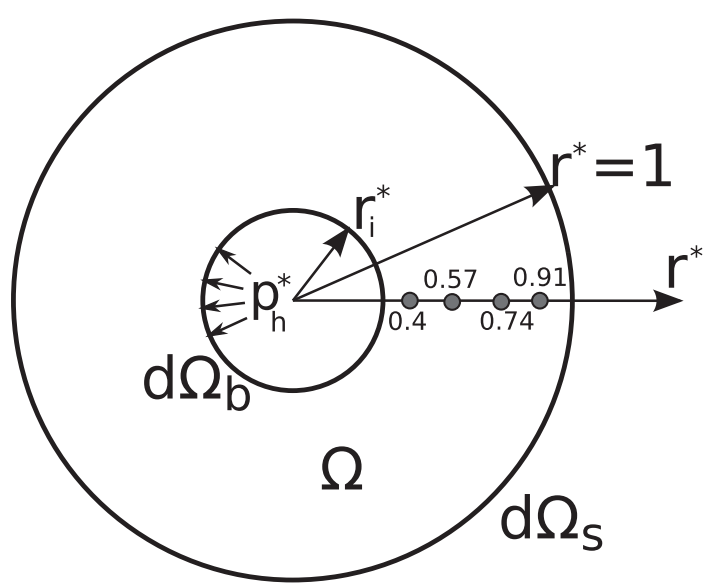

FIGURE 9 Geometry of the borehole injection setting. The outer boundary $d \Omega_{S}$ at $r^{*}=1$ is mechanically free, with zero fluid pressure. The borehole surface $d \Omega_{b}$ at $r^{*}=r_{i}^{*}$ is also mechanically free, but with a fluid pressure $p_{h}^{*}$ caused by fluid injection. The pressure at the 4 points along $r^{*}$ is studied in more detail in Figure 10
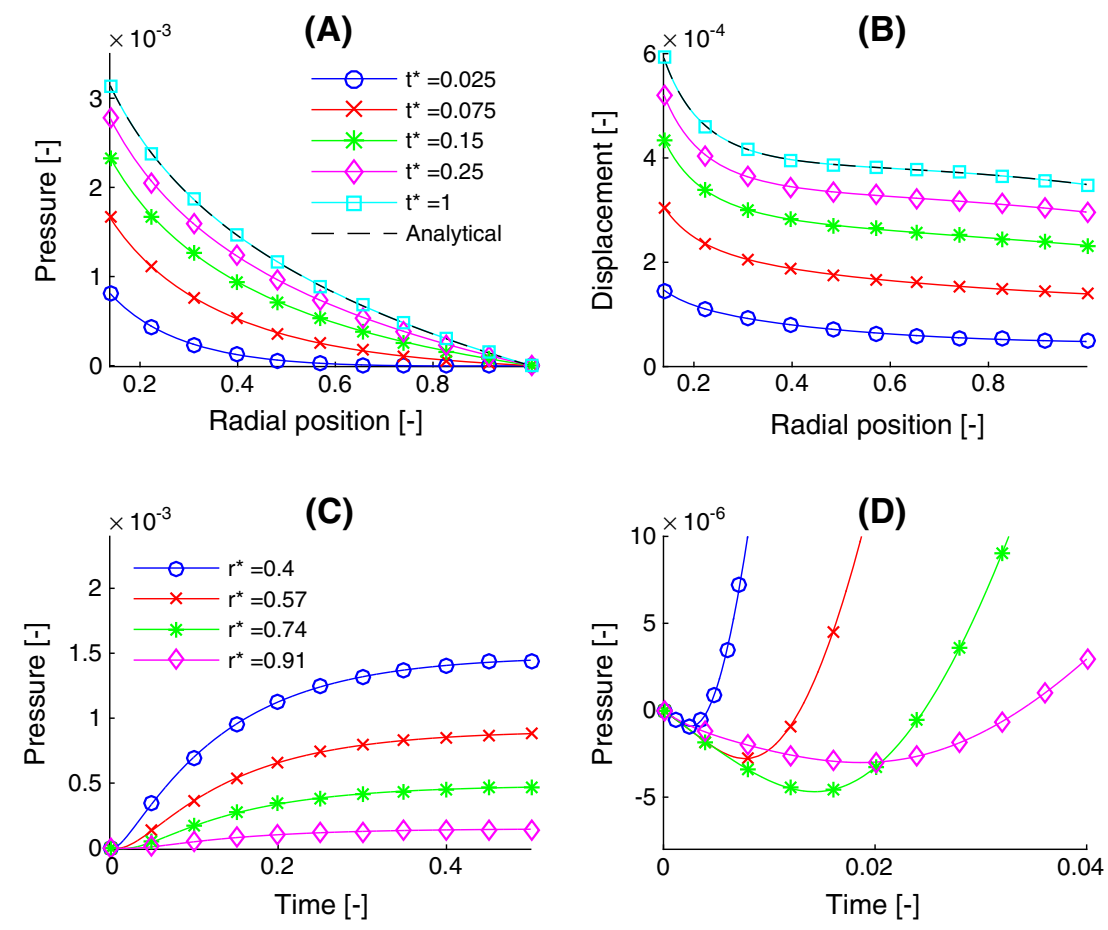

FIGURE 10 Pressure and displacement from 1D model, $b=0.5, Q=0.01$. A, Pressure distribution at 5 different times, with analytical pressure from Equation B3. The pressure increases first near the borehole, and later closer to the rim. Notice that the pressure at $t^{*}=1$ fits the analytical steady state solution well. B, Displacement at 5 different time steps, legend as in A. The displacement increases immediately in the entire domain when the injection starts and increases monotonously. Notice that the analytical steady state displacement from Equation B6 fits the displacement at $t^{*}=1$ well. C, Pressure development in time in 4 different points. The plots show the expected convergence to steady state for a diffusion process. D, Zoom on the pressure development at the start of the injection for the curves shown in C, where the initial response is a negative pressure. Closest to the borehole the injected fluid prevents a negative pressure after a short time, while the negative pressure persists for a longer time further away form the borehole and even becomes increasingly negative. Near the outer boundary, the negative pressure is limited by a backflow from fluid outside the domain [Colour figure can be viewed at wileyonlinelibrary.com]

of steady state pressure in $t^{*} \approx 0.22$, while it takes $t^{*} \approx 0.55$ and $t^{*} \approx 0.5$ for $b=0.5$ and $b=1.0$, respectively. This time is independent of $Q$; with other parameters kept constant, the profiles of pressure divided by $Q$ are identical for different $Q$ at each time step. 
Re-dimensionalizing the time for $b=0.5$ using the parameters from Table 1 shows that constant injection into a typical sandstone $\left(k=10^{-14} \mathrm{~m}^{2}\right)$ with $R=1 \mathrm{~m}$ reaches steady state in just 5 seconds. On the other hand, reaching steady state in the same setting in a tight rock $\left(k=10^{-20} \mathrm{~m}^{2}\right)$ takes nearly 58 days, and the second-order dependence on $R$ means that injection into tight rocks of sufficiently larger size will require years to reach steady state. Thus, injection into tight rocks of sufficient size will in practice never reach steady state in an industrial setting. As a result of this, the transient hydromechanical coupling effect must be taken into account in the engineering analysis.

As the pressure increases, the displacement increases monotonously in time (Figure 10B). Although the pressure distribution at similar borehole pressure does not depend strongly on $b$, the radial displacement shows a significant $b$-dependence. As previously shown, ${ }^{26}$ the radial displacement is concentrated near the borehole for low $b$, while higher $b$ causes stronger interaction between the fluid and solid, and thus also a significant deformation further away from the borehole. This effect is seen at all intermediate stages. The same dependence on $Q$ is seen as for $p$ : Displacement divided by $Q$ is identical at all times for different $Q$.

As seen in Figure 10C, the pressure at the 4 points defined in Figure 9 increases rapidly early in the injection process, before converging more slowly to the steady state pressure, as expected in a diffusion process. However, the pressure is not increasing monotonously for nonzero $b$. Immediately after the injection starts, the pressure goes down (Figure 10D) for some time, before increasing towards steady state. This is caused by a positive volumetric strain: There is a positive displacement far from the borehole (Figure 10B, $t^{*}=0.025$ ) before the pressure front arrives (Figure 10A, $t^{*}=0.025$ ). This is an example of a Mandel-Cryer effect, ${ }^{72,73}$ although with opposite sign of what is usually seen.

The Mandel-Cryer effect is a hydromechanical coupling mechanism in which it is usually seen that when a fluid filled porous material is compressed, fluid flow inside the permeable solid may lead to a gradual pore pressure increase as the initial response of the fluid before the pressure goes down to the level of ambient pressure. This Mandel-Cryer effect depends on the stiffness and permeability of the porous media and the loading rate. In the borehole injection problem, the fluid pressure applied in the borehole instead leads to expansion of the material, and thus a pressure drop at some distance from the injection point.

More detailed studies show that the pressure drop is caused by 2 effects. Firstly, the borehole pressure increases in time and causes increasing volumetric strain near the injection point, which directly leads to a larger pressure drop. However, the transient decrease in pressure is also seen when applying a constant pressure boundary condition in the borehole. The initial response to a pressure applied in the borehole is an expansion in radial direction, which is limited by a tangential total stress (ie, traction along the borehole). This is the total stress, according to Equation 1 equal to a sum of the effective stress and a term related to the fluid pressure. Thus, the deformation is limited by a tensile effective stress and the negative fluid pressure multiplied by the Biot coefficient. As fluid infiltrates near the borehole, the negative pressure is gradually removed, thus transferring more of the total stress to the effective stress and causing even more deformation closer to the rim of the domain. This is more or less exactly what is seen in a Mandel-Cryer effect during compression, but since the material is under tensile stress instead of compression, the sign is reversed, and the pressure becomes negative for a short time.

The magnitude of all effective stress components (Figure 11A-C) and the coulomb stress (Figure 11D) increases in time in response to the increased displacement. The tangential, axial, and coulomb stresses are always positive and increase monotonously in time. High $b$ causes larger stresses away form the borehole, but the stress profiles are otherwise similar. The radial stress shows somewhat more complicated dynamics and starts out as compressive in the entire domain at the start of the injection, before becoming tensile in a region away from the borehole near steady state. In the end points, the region of positive stress is nonexistent for $b=0.0$ and fills the entire domain for $b=1.0$. As for pressure and displacement, the time development of all stresses divided by $Q$ is independent of $Q$ at all times when other parameters are kept constant.

The dominating stress component is always found to be the tangential stress, which is highest at the wall of the borehole, and increases in time until reaching steady state. Thus, the fracture that might be formed if $Q$ is sufficiently high is tensile, initiates at the borehole wall and grows initially in the radial direction. Shear fractures will not form initially, unless the rock is unusually weak in compression, with $C_{u} / T_{u} \lesssim 5.5$. The following fracture growth is studied in more detail in Sections 3.3 and 3.4.

\section{3 | Fracture development in high-permeability rock}

In this section, we study the potential fracture formation caused by continuous fluid injection into the borehole that was studied in the previous section. The geometry and boundary conditions are presented in Figure 9 . Here, we present results for high-permeability rocks with $k=10^{-14} \mathrm{~m}^{2}$, before turning to low-permeability rocks in the next section. 

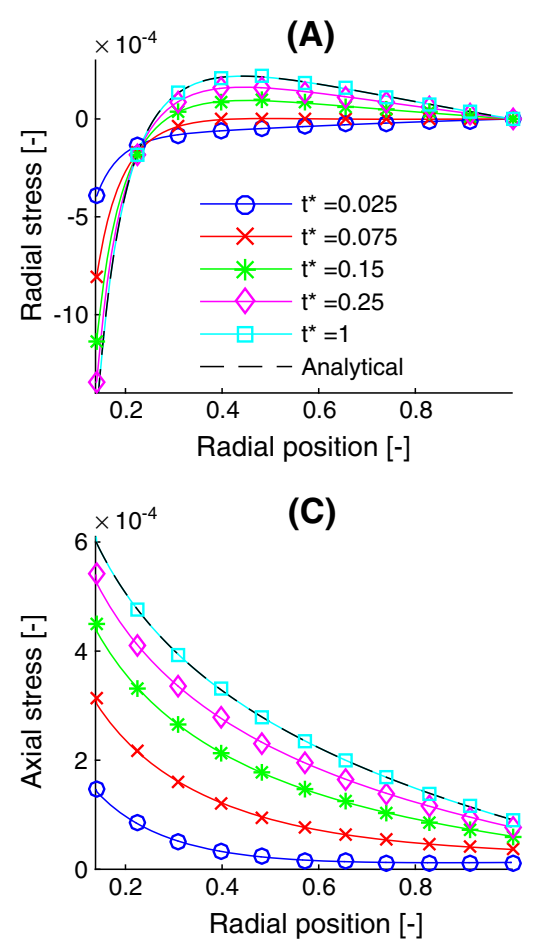

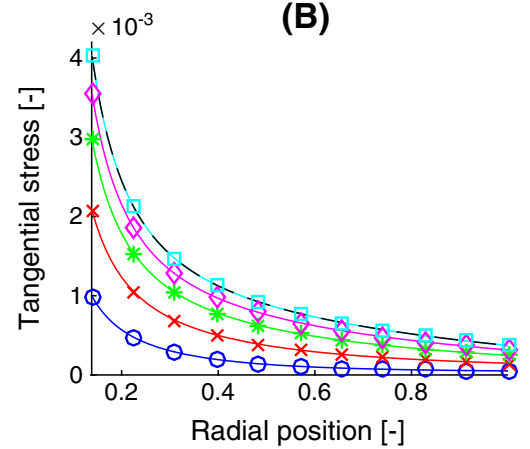

(D)

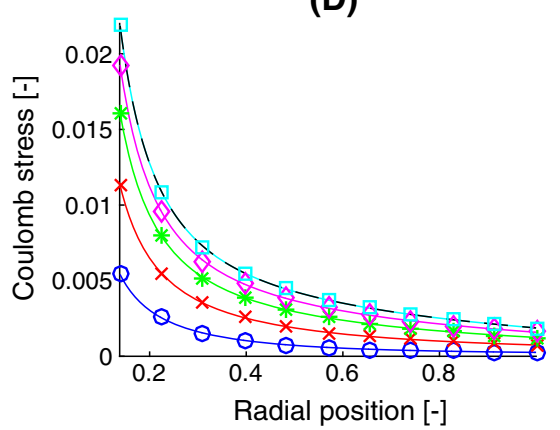

FIGURE 11 Stress development in time for the 5 time steps for which pressure and displacement are shown in Figure 10. The legend for all 4 plots is shown in A, and analytical solutions for stress components (A-C) from Equations B7 to B9 and for the coulomb stress from Equation 11 fit the final stress state well. A, The radial stress is initially compressive in the entire domain but gradually becomes tensile near the outer boundary for nonzero $b$. B. The tangential stress is always positive, is the dominating stress component, and increases monotonously in time. $\mathrm{C}$, The axial stress is also tensile and increases in time but is generally nearly an order of magnitude smaller than the tangential stress. $\mathrm{D}$, The coulomb stress is always positive and increases monotonously in time but is not large enough to make the rock fail in compression before it fails in tension, unless the rock is unusually weak in compression [Colour figure can be viewed at wileyonlinelibrary.com]

Fracture formation is expected to affect the unfractured process in the previous section in 2 ways. Firstly, fractures obviously allow increased mechanical displacement. This can cause increased expansion of the borehole, which lead to a borehole pressure drop, and cause formation of open volumes available for inflow of fluid in the bulk material. Secondly, fractures are likely to have higher permeability than the bulk rock and thus lead to more efficient drainage of the injected fluid.

In the first set of simulations, we focus on the increased displacement and ignore the potentially significant permeability increase in fractures compared to bulk rock. Thus, $k_{f}=10^{-14} \mathrm{~m}^{2}$. Figure 12 shows snapshots from the pressure and fracture development for $b=0.2$ and $b=0.8$ when a moderate injection rate $Q=0.01$ is used. In both cases, the pressure increases near the borehole, and radial fractures initiate at the borehole wall, as predicted in the previous section. The fractures continue to grow radially in time until reaching the domain boundary, while the pressure increases mostly monotonously and converges to a stable pressure after a long time. Even without additional fluid transport in fractures, the stable pressure is lower than in the unfractured case. The most striking difference between the fracture development for different Biot coefficient is the fracture density, with 3 nearly equidistant fractures for $b=0.2$, and 7 fractures for $b=0.8$.

While the borehole pressure increases in time for both Biot coefficients, there is a negative fluid pressure of the same order of magnitude that develops in and along the fractures. This is caused by the open volume formed in the fractures: Without any additional fluid transport in the fractures, sufficient fluid cannot be supplied. In this case, it represents a viscous damping of the deformation. Even when all fractures reach the outer boundary and the material is mechanically free to expand outwards, the negative fluid pressure will limit the deformation. A pressure balance is set up: The positive pressure in the borehole tries to expand the material, while the negative pressure prevents the expansion. A higher Biot coefficient means that the negative pressure prevents expansion more efficiently, and thus, the borehole pressure when the fractures have reached the boundary is highest for $b=0.8$, while the negative pressure in the fractures is similar to the situation for $b=0.2$. This force balance defines the steady state pressure in the fractured case and causes a lower steady 

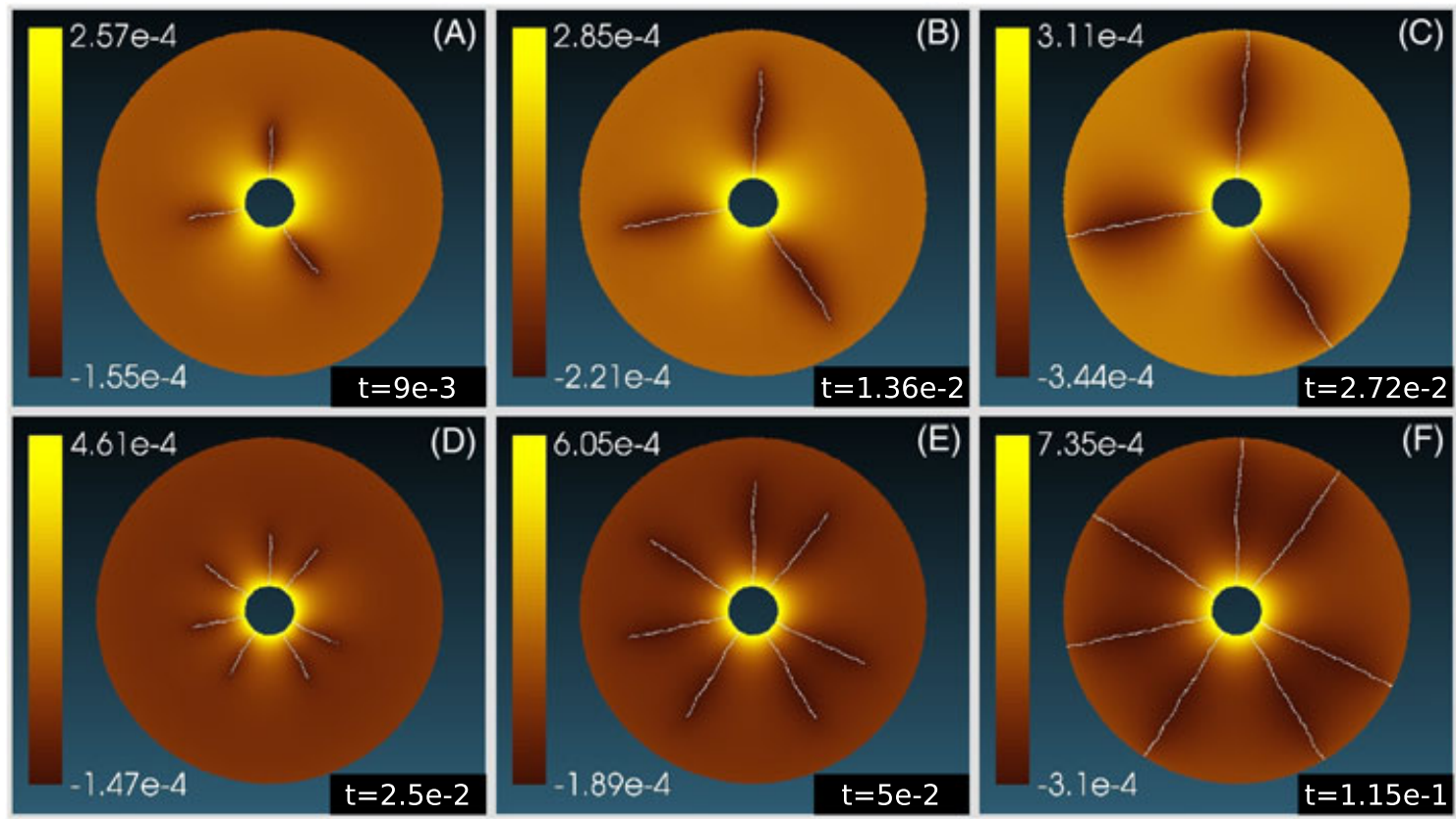

FIGURE 12 Discrete element model results for $Q=0.01, l=1$ at 3 time steps, A-C: $b=0.2$, D-F: $b=0.8$, with scale showing dimensionless fluid pressure $p^{*}$. The pressure limits reflect the maximum and minimum pressure present in each case. A, Three radial fractures have initiated. Notice that insufficient fluid in the fractures causes a negative pressure. B, The fractures continue to grow radially outwards. Both the positive pressure in the borehole and the negative pressure in the fractures have increased in magnitude. C, The fractures have reached the domain boundary. The negative pressure in the fractures cause draining of fluids from the porous material along fractures, thus giving a zone of negative pressure. Near the domain boundary, the negative pressure is cancelled by backflow from the outside. D, Seven radial fractures have initiated. E, All fractures continue to grow, and the borehole pressure is increased. As for $b=0.2$, there is a negative pressure inside the fractures. F, All fractures have reached the domain boundary. Notice that all fractures are almost exactly radial [Colour figure can be viewed at wileyonlinelibrary.com]

state pressure than in the unfractured case. Furthermore, it is clear that there is no steady state mechanical configuration, since the material will continue to expand indefinitely when more fluid is injected.

Figure 13 shows the development of radial displacement for the 2 cases above. Before fractures are formed, the displacement from DEM follows 1D results closely, but differences occur when fractures form. Then the borehole expands slightly, and the borehole pressure increases more slowly than in the unfractured case. For $b=0.2$, the displacement immediately increases at all radial positions and continues to increase in time. On the other hand, for $b=0.8$, the displacement increases in the fractured zone only, while the displacement far from the borehole actually is lower than in the unfractured case. For high $b$, the material expansion is limited by the negative pressure that is caused by a volume expansion, while low $b$ gives a weaker coupling and allows more displacement. The reduced borehole pressure thus counterintuitively causes less displacement for high $b$ in the unfractured zone than in the completely unfractured case. Even when the fractures reach the boundary, there is little additional deformation far from the borehole for high $b$.

This case is revisited in Section 3.6 for the average value $b=0.5$, where we show that the effect of changing grid resolution is minor above a minimum resolution.

When fluid transport in fractures is ignored, a negative fracture pressure limits the fracture growth rate. This changes significantly when fluid is allowed to flow faster in the fractures. Figure 14 revisits the setting from Figure 12, but now with $k_{f}=10^{-11} \mathrm{~m}^{2}$, thus giving 3 orders of magnitude faster fluid transport in fractures than in the bulk rock. When the first fractures form, the accelerated fluid flow immediately causes the borehole pressure to drop. This reduces the number of fractures that are initiated, but at the same time, the fracture growth rate is increased. Thus, faster infill of fluid in the fractures allows more rapid widening of the fracture aperture, which causes higher stress at the fracture tip, and ultimately causes the fractures to reach the boundary faster for both high and low $b$ than in the previous case. The steady state is similar to the previous case, but efficient drainage of fluid leads to a lower steady state pressure, which in turn causes slower expansion of the material.

The radial displacement development with $\imath=10^{3}$ shows some differences from the case without additional fluid transport (Figure 15). Notably, the pressure drop caused by efficient drainage of fluid in the fractures causes less deformation 

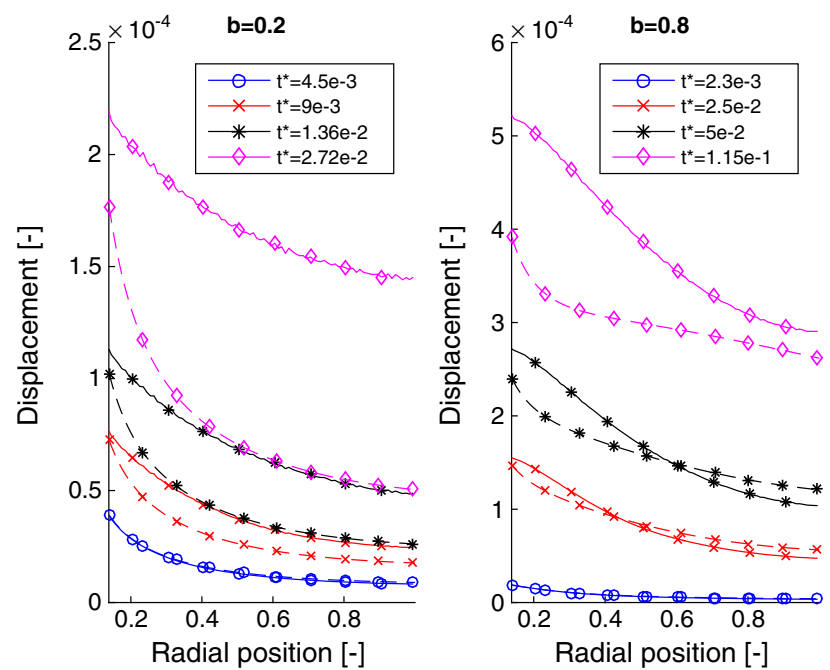

FIGURE 13 Average radial displacement as function of radial position for the discrete element model (DEM) cases shown in Figure 12. Solid lines show data from DEM, and dashed lines show 1D finite difference model (FDM) results for the same time step without fracture formation. Left panel: $b=0.2$. At $t^{*}=4.5 \cdot 10^{-3}$, there are no fractures, and DEM data fit 1D FDM data. At $t^{*}=9 \cdot 10^{-3}$, fractures have initiated, and the displacement increases at all radial positions compared to the situation without fractures. When the fractures reach the boundary at $t^{*}=2.72 \cdot 10^{-2}$, the displacement increases sharply, especially near the boundary. Right panel: $b=0.8$. The DEM data fit the $1 \mathrm{D}$ FDM data nicely when there is no fractures at $t^{*}=2.3 \cdot 10^{-3}$. When fractures have initiated at $t^{*}=2.5 \cdot 10^{-2}$, the displacement increases in the fractured area, but at the same time, the displacement is reduced at larger radial positions. The same trend continues at $t^{*}=5 \cdot 10^{-2}$, and when the fractures reach the boundary at $t^{*}=1.15 \cdot 10^{-1}$, the displacement increases markedly near the borehole, but less near the boundary [Colour figure can be viewed at wileyonlinelibrary.com]
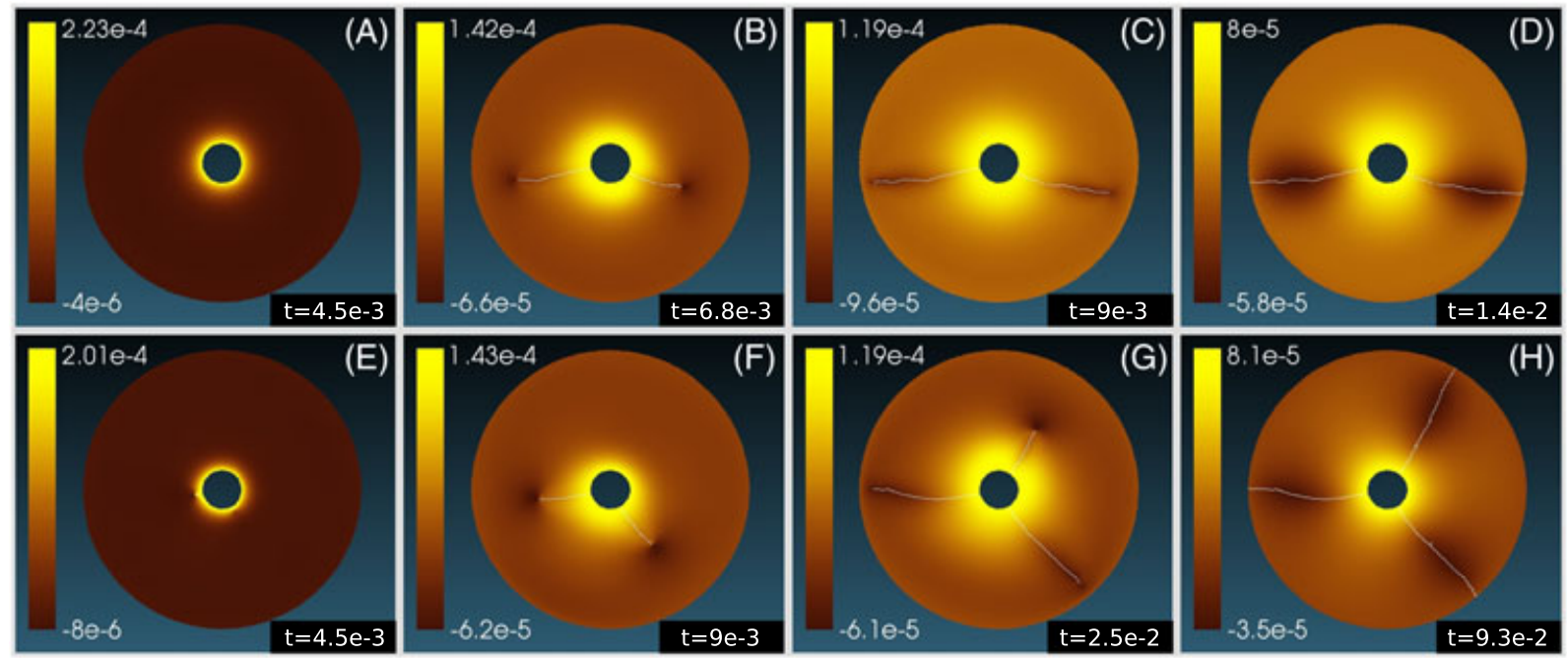

FIGURE 14 Discrete element model results at 4 different time steps for $Q=0.01, l=1000$. A-D: $b=0.2$, E-H: $b=0.8$. The scale bar shows dimensionless fluid pressure $p^{*}$. A, There is a high-pressure region near the borehole, and the Mandel-Cryer effect causes a negative pressure further away from the borehole. B, Fractures have initiated. The borehole pressure has dropped significantly, and there is a zone of negative pressure ahead of each fracture tip. C, The fractures have almost reached the boundary. D, Both fractures have reached the boundary, and rapid fluid flow causes a pressure drop. The deformation is now limited by a negative pressure in the fractures, as was also seen in the case without enhanced fluid transport in the fractures. E, There is a high-pressure region near the borehole, and 2 hardly visible fractures have initiated. F, The fractures continue to grow, and there is a negative pressure ahead of the fracture tips. G, A third fracture is initiated. H, All 3 fractures have reached the boundary, and the pressure is reduced [Colour figure can be viewed at wileyonlinelibrary.com]

near the borehole, while the deformation near the borehole increased without the enhanced fluid transport. At the same time, the rapid infill of fluid in the fractures reduces the negative pressure seen in the previous case and allows a more uniform expansion at all radial positions. 

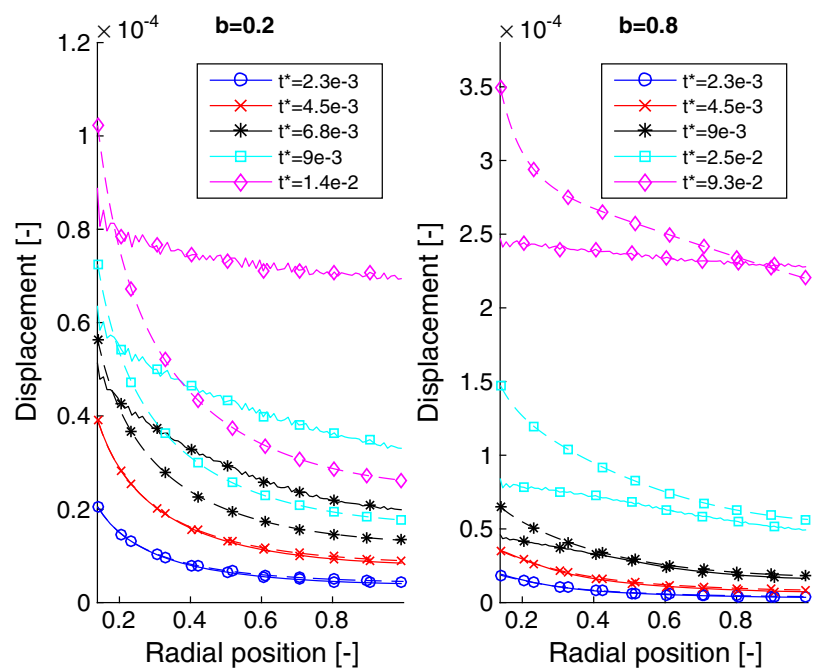

FIGURE 15 Average radial displacement as function of radial position for the discrete element model (DEM) cases shown in Figure 14. Solid lines show data from DEM, and dashed lines show 1D FDM results for the same time step without fracture formation. Left panel: $b=0.2$. At $t^{*}=2.3 \cdot 10^{-3}$ and $t^{*}=4.5 \cdot 10^{-3}$, there are no fractures, and DEM data fit 1D FDM data. At $t^{*}=6.8 \cdot 10^{-3}$, fractures have initiated, and the displacement increases at most radial positions compared to the situation without fractures, but a pressure drop in the borehole reduces the displacement near the borehole. This development continues, and when the fractures reach the boundary at $t^{*}=1.4 \cdot 10^{-2}$, the displacement is almost constant through the domain. Right panel: $b=0.8$. The DEM data fit the 1D FDM data nicely when there are no fractures at $t^{*}=2.3 \cdot 10^{-3}$ and $t^{*}=4.5 \cdot 10^{-3}$. When fractures have initiated at $t^{*}=9 \cdot 10^{-3}$, the displacement is reduced at all radial positions. The same trend continues at $t^{*}=2.5 \cdot 10^{-2}$, and when the fractures reach the boundary at $t^{*}=9.3 \cdot 10^{-2}$, the displacement is nearly constant throughout the domain, but larger than in the unfractured case only near the boundary [Colour figure can be viewed at wileyonlinelibrary.com]
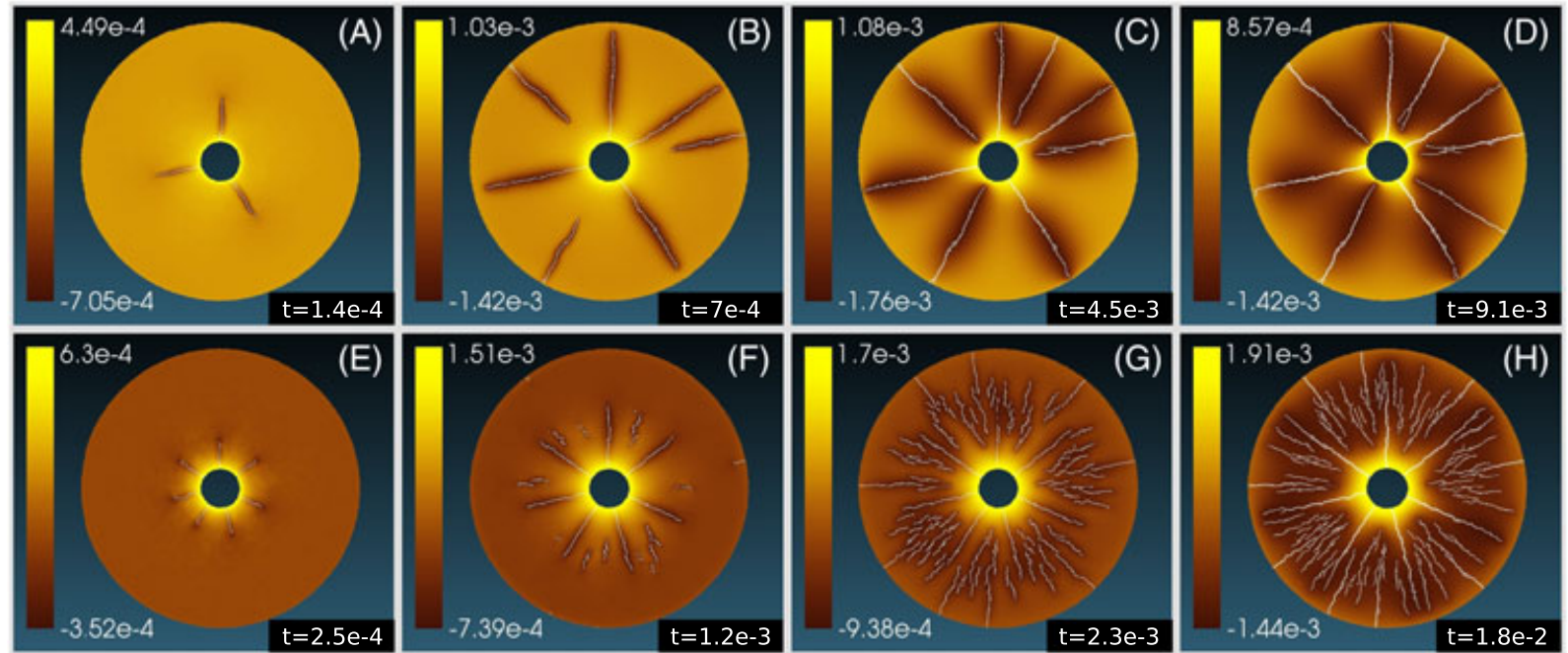

FIGURE 16 Discrete element model results at 4 different time steps with $Q=1, l=10^{3}$. A-D: $b=0.2$, E-H: $b=0.8$. The scale bar shows dimensionless fluid pressure $p^{*}$. A, Three radial fractures have initiated. The fractures open too rapidly to become fluid filled, thus causing a negative pressure. B, Four radial fractures are growing towards the domain boundary, and secondary fractures initiate at the domain boundary and grow towards the borehole. $\mathrm{C}$, The outwards growing fractures have nearly reached the domain boundary, and the inwards growing fractures have nearly reached the borehole. A high pressure in the borehole is balanced by a high negative pressure in the fractures. $\mathrm{D}$, One outwards growing fracture reaches the boundary, and the borehole pressure drops. E, Seven radial fractures have initiated. F, Secondary fractures initiate between the growing radial fractures. G, There is a dense formation of unconnected secondary fractures between the primary fractures growing from the borehole, and the pressure continues to increase. $\mathrm{H}$, The pressure increases further, and the primary fractures are almost connected to the boundary. Several secondary fractures are connected to the boundary, causing a backflow of fluid into the domain [Colour figure can be viewed at wileyonlinelibrary.com] 
When the injection rate is increased, the fracture development becomes significantly more violent. An example of this is shown in Figure 16, where $Q$ is increased to 1 . Notice that the initial fracture formation in this case is almost the same as seen in Figure 12. The fluid is injected in the borehole faster than the fractures are able to drain fluid, and the pressure in the borehole thus increases more than in the $Q=0.01$ case. The high borehole pressure opens the fractures faster than they are filled by fluid and causes a negative fracture pressure. While low $Q$ leads to fracture formation driven by a high pressure in the fractures, higher $Q$ gives fracture formation that is driven by the borehole pressure and limited by a negative pressure in fractures.

High $Q$ also causes a secondary fracture formation that was not observed in the low $Q$ case. The secondary fractures show a significant $b$-dependence: For low $b$, secondary fractures initiate at the domain boundary, while for high $b$, the secondary fractures initiate at lower $r^{*}$ between the initial fractures. The reason for this behavior can be seen in Figure 13. For $b=0.2$, the radial displacement increases at all radial positions when fractures initiate near the borehole. This promotes fracture formation at the domain boundary. On the other hand, for $b=0.8$, the radial displacement increases near the borehole when fractures initiate, but not closer to the boundary. Thus, secondary fractures initiate between the primary fractures.

In the absence of mechanical confinement, the borehole continues to expand indefinitely. This leads to a nearly uniform negative pressure in a zone between the borehole and the domain boundary, while a high pressure near the borehole drives the deformation, and a backflow from the boundary gives a pressure near zero in a zone near the boundary.

\section{4 | Fracture development in low-permeability rock}

In this section, we focus on borehole injection in low-permeability rocks with $k=10^{-17} \mathrm{~m}^{2}$. If all dimensionless parameters are kept constant, there is no reason to expect different results from low-permeability rock, apart from a change in characteristic time scale. However, the permeability in a fracture might be the same in a low-permeability rock as in a high-permeability rock. Thus, we keep $k_{f}=10^{-11} \mathrm{~m}^{2}$ as in the previous section, which gives $\iota=10^{6}$.

Figure 17 shows an example of a low-permeability rock with $Q=1, l=10^{6}$. The low permeability means that a significant part of the domain is unaffected by fluid flow, and fluid is drained through fractures instead. In the final fracture configuration (Figure 17D,H), there is a high-pressure zone near the borehole and along part of the fractures and a thin layer near the boundary with pressure near zero, but in the remaining material, there is a negative pressure caused by the Mandel-Cryer effect discussed in Section 3.2. The flow is clearly far from equilibrium, although the fractures drain the injected fluid sufficiently fast to prevent further fracture formation.

The main pressure fluctuations in the low permeability case are caused by mechanical deformation. In a small zone ahead of fracture tips, the material expands slightly and causes a localized negative pressure. A similar behavior was seen in Figure 14. It is also seen (Figure 17B) that volumetric strain around the borehole can cause a zone of negative pressure, which will draw fluid from the borehole. When the strain is released by a new fracture (Figure 17C), the additional fluid drawn into the low-pressure zone causes a high-pressure zone instead. This high-pressure plume remains for a long time in a low-permeability rock, and it is seen in Figure 17D that fluid flows back into the borehole, before escaping through fractures.

\section{5 | The borehole breakdown pressure}

The borehole breakdown pressure, the pressure at which tensile failure occurs around a borehole, is of central interest to determine the in situ stress field in a rock mass. In this section, we focus on the breakdown pressure in permeable rocks.

Figure 18 shows the pressure recorded at fracture initiation for a range of different injection rates and for different Biot coefficients. At low injection rates, the borehole breakdown pressure is below the dimensionless tensile strength of the rock. For significantly lower injection rates, there is no fracture formation, and thus no breakdown pressure can be recorded. As $Q$ increases, the breakdown pressure increases almost linearly as a function of $\log _{10}(Q)$ until it starts to level off above the dimensionless tensile strength of the rock. This effect is stronger for high $b$.

\subsection{Effects of lattice beam size}

In this section, we conduct a refinement study to quantify how the length of the lattice beams affect the macroscopic outcome. In particular, we revisit the first setting from Section 3.3 assuming $b=0.5, Q=0.01$ and $l=1$. For this purpose, we choose a range of grid resolutions where the domain is filled with from 684 particles in the coarsest grid to 170158 particles in the finest counterpart. 

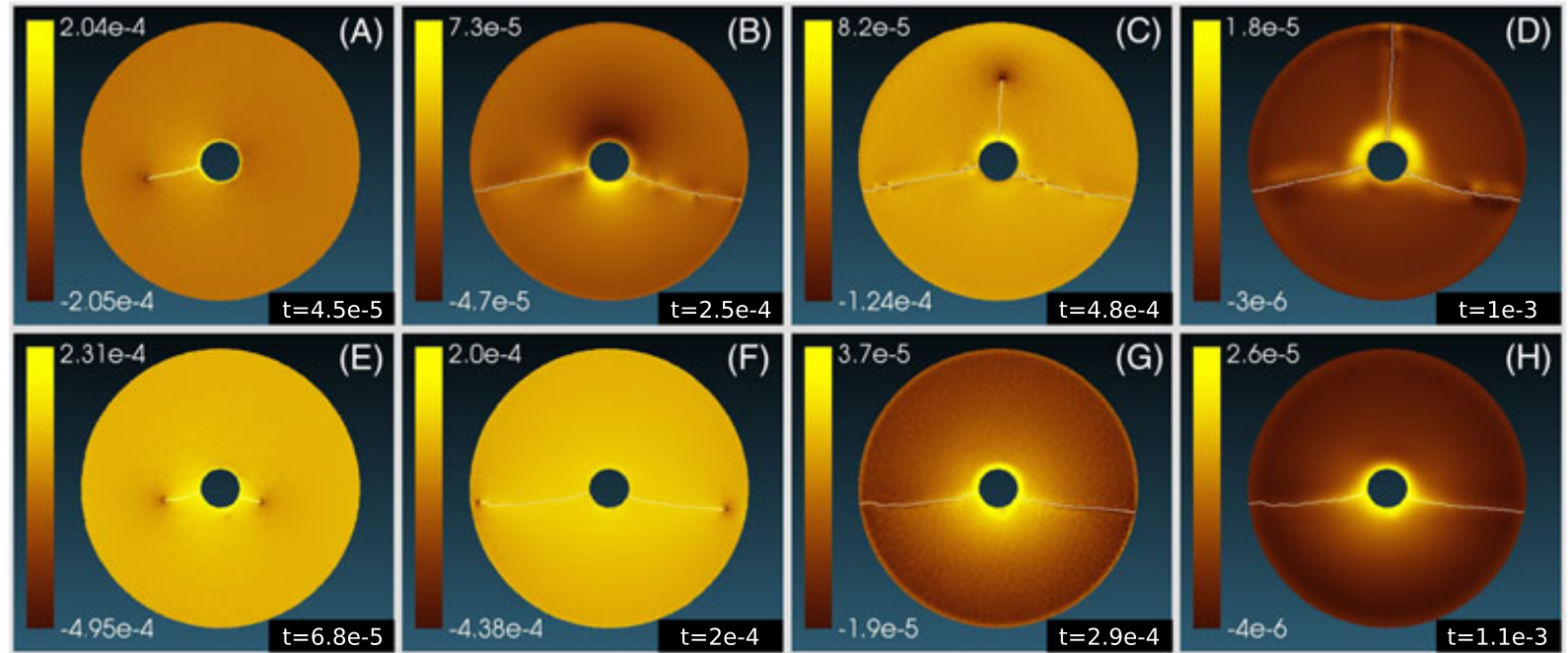

FIGURE 17 Discrete element model results at 4 different time steps for a low-permeability rock, $Q=1, l=10^{6}$. A-D: $b=0.2$. E-H: $b=0.8$. The scale bar shows dimensionless fluid pressure $p^{*}$. A, There is a thin high-pressure region around the borehole, and a single radial fracture is growing. Notice the region of negative pressure ahead of the fracture tip. B, Two radial fractures are connected to the boudary, and the pressure is reduced. A positive volumetric strain above the borehole leads to a significant negative pressure. C, A third radial fracture is initiated. Notice that the region of negative pressure in panel B is now the region with highest pressure. D, All 3 fractures are connected to the boundary and drain the injected fluid efficiently. The low-pressure region from panel B remains the region with highest pressure, and fluid flows back into the borehole. E, Two radial fractures have initiated. F, The fractures have almost reached the boundary. Notice that there is a narrow region of high pressure around the borehole and along the fracture, and a high negative pressure ahead of the fracture tip. G, The pressure scale changes significantly when the fractures reach the boundary. $H$, The borehole pressure is significantly reduced. The high-pressure region around the borehole remains for a long time and leads to backflow into the borehole. A positive volumetric strain away from the borehole causes a slightly negative pressure [Colour figure can be viewed at wileyonlinelibrary.com]

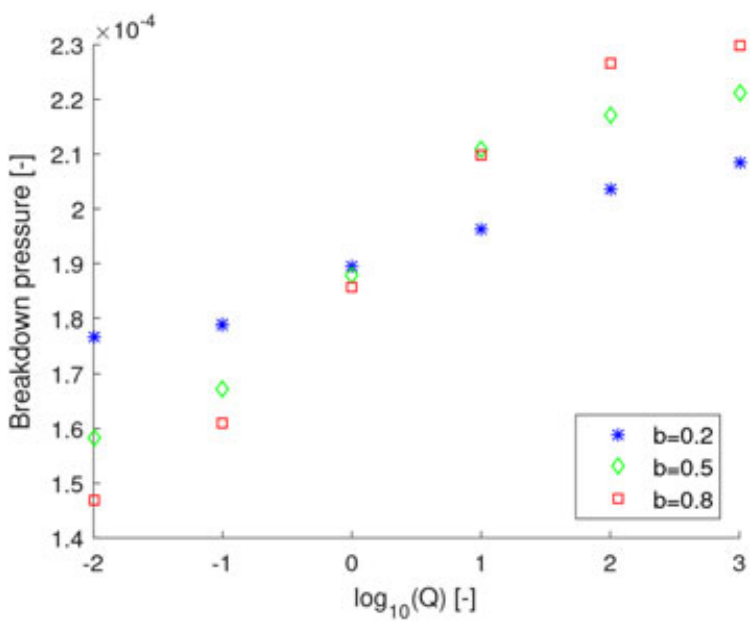

FIGURE 18 Dimensionless borehole pressure at fracture initiation as function of dimensionless injection rate $Q$ for different Biot coefficient. Notice that the breakdown pressure is below the dimensionless tensile strength of the rock, $T_{u} / E=2 \cdot 10^{-4}$, for low injection rates, and above the dimensionless tensile strength for high $Q$ [Colour figure can be viewed at wileyonlinelibrary.com]

Snapshots of fractures and pressure distribution at time $t^{*}=2.5 \cdot 10^{-2}$ are shown in Figure 19. For all lattice sizes, there is a similar high-pressure region near the borehole, and a halo of negative pressure around the fractures. Apart from panel A, which has the lowest resolution, all panels show 4 fractures, similar fracture lengths and similar maximum pressure. The minimum pressure varies significantly in time and drops markedly when a fracture grows and creates new space. Accordingly, the differences in the recorded minimum pressure between panels B to $\mathrm{F}$ are within the normal variation in time. Due to axisymmetry in the simulated scenario, the orientation of the fractures is random and varies between the different simulations. The same behavior is seen when we compare simulations using different grids at the same resolution. 

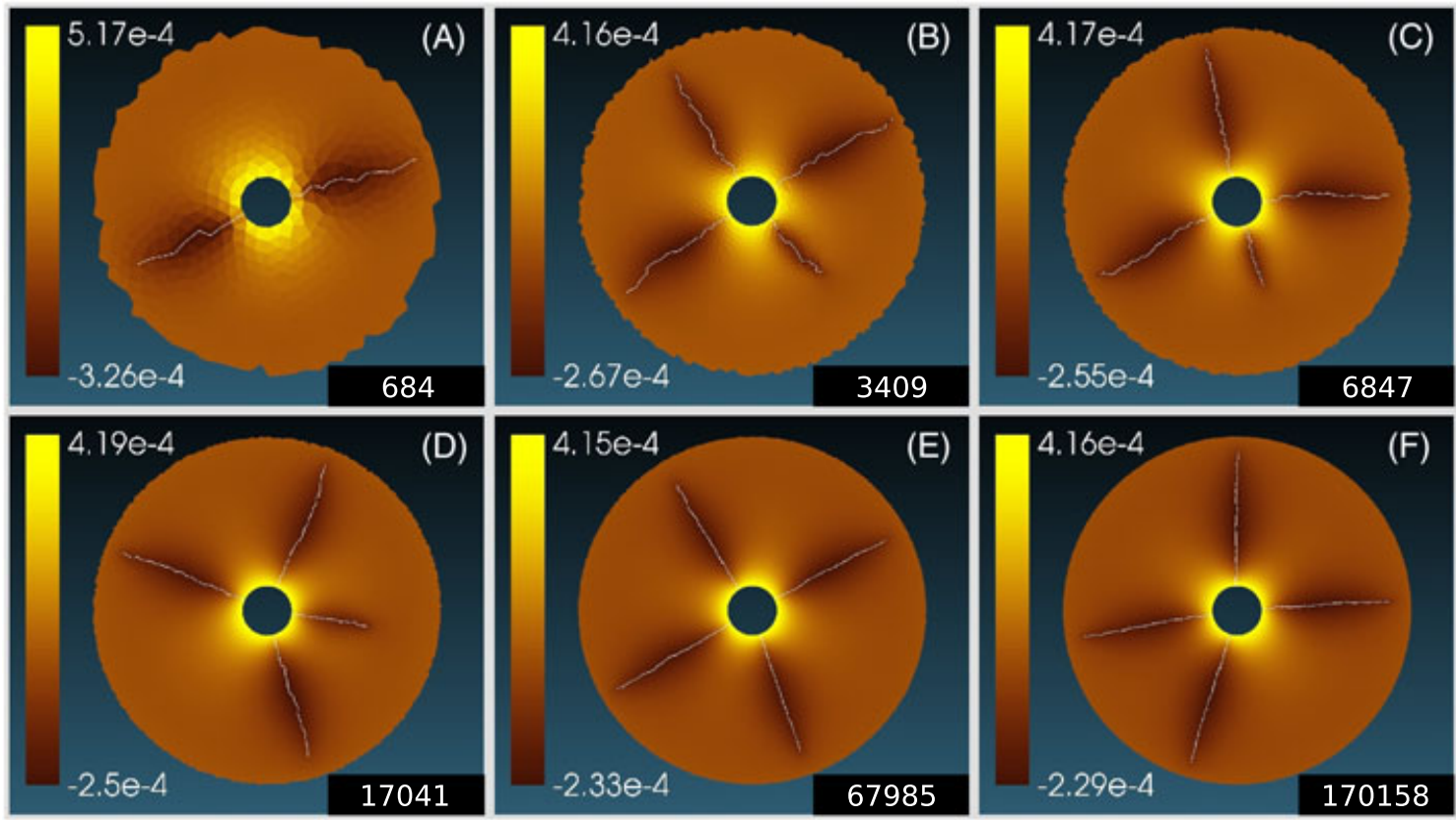

FIGURE 19 Discrete element model results for $Q=0.01, l=1$ at time $t^{*}=2.5 \cdot 10^{-2}$ using 6 different grid resolutions, with number of particles shown in lower right corner. With the lowest resolution in A, only 2 fractures are formed, while all simulations with higher resolution lead to 4 fractures. Fractures are located randomly due to rotational symmetry, but are relatively evenly spaced, and most fractures are of similar length. Notice that the pressure range is similar for all cases with 4 fractures, but that the pressure range is larger when only 2 fractures are present [Colour figure can be viewed at wileyonlinelibrary.com]

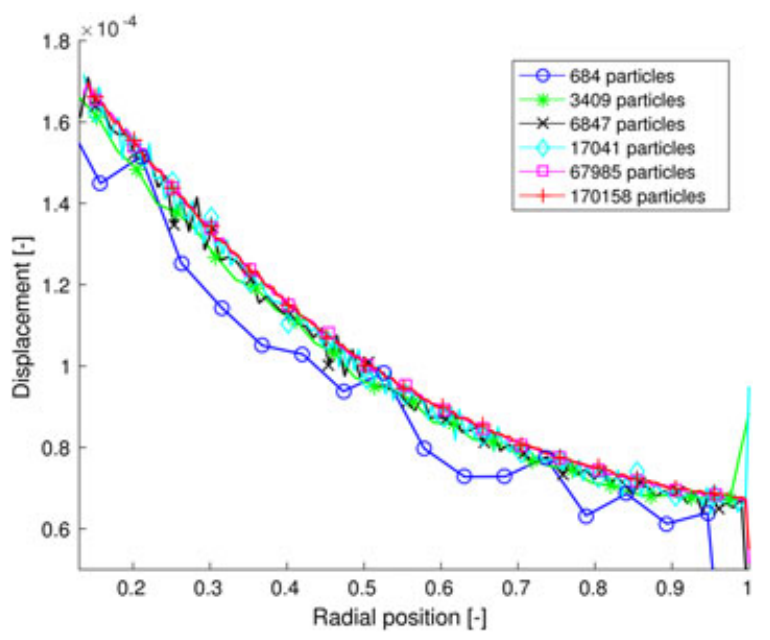

FIGURE 20 Average radial displacement as function of radial position for the 6 different grid resolutions shown in Figure 19. Notice that the 5 highest resolutions produce almost identical displacement, while the lowest resolution simulation give noticeably lower radial displacement [Colour figure can be viewed at wileyonlinelibrary.com]

Figure 20 shows the radial displacement for the 6 different grid resolutions, averaged over radial positions. Apart from some random deviations, the 5 simulations with highest resolution show similar deformation, while the lowest resolution, where only 2 fractures are present, predicts lower displacement.

\section{4 | DISCUSSION}

In this work, we have introduced a two-way hydromechanical coupling between a fluid flow solver and a solver for mechanical deformation. The benchmark, using analytical solutions for fracture aperture and point injection, shows that 
this coupled solver captures the physics of fluid flow and deformation well, as seen in Section 3.1 and Appendix C. Further, it is clear that the undrained operator split with an optimal coupling parameter $\Psi$ performs significantly better than the drained split in this case. The results given in Sections 3.2 to 3.4 highlight the importance of this process coupling for borehole injection at constant injection rate.

Our dual-grid approach follows closely the method introduced by Grassl et al, ${ }^{26}$ but where they assumed that the fluid flow was always in equilibrium, we have focused on the transient behavior during continuous fluid injection. Part of the reason for this choice can be seen in Section 3.2. Especially for a low-permeability rock, reaching steady state takes a long time. To avoid significant deviation from a steady state pressure distribution, the injection rate thus needs to be increased slowly. We regard this as less relevant for industrial purposes.

Another reason for including the full transient setting is that fracture formation is a path and history-dependent process. Fractures do not only affect the stress field but also create void space that requires infill of fluids and form channels that might transport fluids more efficiently. Even when changing the injection rate slowly, the pressure evolution in a growing fracture might be far from equilibrium. As seen in numerical examples in this paper, the fluid pressure in a fracture might drop below the pressure in the intact material nearby, or exceed that pressure, depending on injection rate and how efficient fluid flow in fractures is. For low-permeability rock, an even more striking example is the pressure change caused by variations in volumetric strain. As seen in Figure 17B-C, a positive volumetric strain can cause a low-pressure region that effectively sucks in fluid from the borehole. When a new fracture releases the strain in the material, the same region contains too much fluid and becomes a high-pressure region. Variations in volumetric strain thus acts as a fluid pump. Such pressure variations are rapidly dissipated in high-permeability rock but remain for a long time in low-permeability rock.

A refinement study is conducted in Section 3.6 to examine the effect of the lattice beam length on the macroscopic responses. Apart from the lowest resolution with only 684 particles, the different resolutions give similar results, although with varying tangential orientation of the fractures due to axisymmetric conditions. We interpret the deviation for the low resolution case as an inability to represent the pressure distribution properly when the length of the lattice beam is sufficiently large compared to the boundary layer of the pore pressure. As a result, the high-pressure region around the borehole is mostly limited to one layer of triangles (Figure 19A). Thus, the fluid pressure interacts mainly with the borehole wall and not the bulk material. This is similar to a simulation with low Biot coefficient $b$, and it is shown clearly in Figure 12 that low $b$ leads to fewer fractures than high $b$.

The results are clearly mesh dependent in the sense that the specific layout of the grid affects the details of the fracture pattern-eg, the fractures have to grow around the particles-but this is also seen when comparing simulations using different grids with the same lattice beam length. This mesh dependence is not unique for the present model. However, for this reason, any trend in the behavior of the system that is caused by different lattice beam lengths is at least too weak to be discerned in the results shown in Figures 19 to 20. Statistics from a large number of simulations at each resolution might reveal a systematic difference in some measurable property, but we regard such a detailed study of the potential mesh size dependence as outside the scope of the present work.

\section{1 | Dependence on injection rate, Biot coefficient, and fracture-enhanced permeability}

For high $Q$ or low $l$, it is seen (Figures 12 and 16) that fluid is injected faster into the borehole than the fractures are able to transport the fluid. This causes a buildup of pressure in the borehole, which may drive further fracture formation. In this setting, the fractures are unable to become fluid filled, and a negative pressure is the result. Although we do not attempt to study fracture tip asymptotics in any detail, the observation of a negative pressure resembles what Detournay ${ }^{68}$ termed large lag. In that case, when fluid did not fill the fracture sufficiently fast, it was assumed that the lag region between the crack tip and the fluid front was filled with the vapors of the fluid, with a negligible pressure compared to the far-field stress. This defines the minimum pressure that might be observed in a fracture, which is $-p_{0}$ in this work. An even lower pressure would clearly lead to vapor cavitation and unsaturated conditions. Although models for unsaturated poroelasticity have been suggested ${ }^{74}$ we regard unsaturated conditions as outside the scope of the present work and have assumed that $p_{0}$ is sufficiently high to allow any negative pressure observed in our simulations. Detournay ${ }^{68}$ did not discuss large lag in permeable rocks in any detail, but this work shows that there will be a significant backflow of fluids from the bulk rock into the fractures (Figure 16). As a practical application, the backflow of fluid might affect the chemistry of fracking fluids in settings with large lag. 
On the other hand, when $Q$ is low or $\iota$ is very high, fluid enters fractures rapidly enough to prevent significant negative pressures (Figures 14 and 17). This resembles the no lag setting, ${ }^{68}$ when the fracture tip and the fluid front coalesce into one combined front. In this case, we see fractures driven both by the pressure in the borehole and by pressure in the fractures, and it causes a negative fluid pressure ahead of the fracture tip where the material is expanding slightly prior to failure. Notice that with zero lag, the fractures are less straight than in the large lag case, with less radial symmetry. In the final state, when fractures reach the boundary of the domain, the lack of mechanical confinement leads to a negative pressure in most fractures far from the borehole independent of $Q$ and $l$, and the mechanically free segments surrounding the borehole are only limited from expanding freely by the negative pressure in fractures.

In Figure 12, it is seen that high $b$ causes higher fracture density than low $b$. Even though higher fracture density for high $b$ might intuitively make sense due to the strong interaction between the solid and the fluid, this is the opposite behavior of what was reported by Grassl et al. ${ }^{26}$ The reason for the results presented in this paper can be seen in Figure 13 . When fractures initiate for low $b$, the radial displacement increases immediately at all radial positions, and the borehole pressure drops. At the same time, the radial displacement leads to a positive volumetric strain that causes a negative pressure far from the borehole. For high $b$, the volumetric strain by definition deforms the fluid to a larger extent, and thus causes a higher negative pressure, which prevents a large displacement far from the borehole. The borehole pressure remains high, and in turn leads to initiation of additional fractures near the borehole.

For a high injection rate and rapid fluid transport in fractures (Figure 16), we see initiation of secondary fractures at the domain boundary for low $b$, and closer to the borehole for high $b$. For low $b$, this can be understood based on Figure 13, where we see that the displacement increases significantly far from the borehole when fractures initiate at the borehole surface. This causes an increased tangential stress near the outer surface of the domain. According to Equation 1, the total stress $\sigma=\sigma_{m}+b \sigma_{f}$ is comprised of a mechanical component $\sigma_{m}$ and a component from the fluid pressure $b \sigma_{f}=-b p I$. Far from the domain surface, the fluid pressure is negative, thus giving a lower $\sigma_{m}$ for a constant $\sigma$. Backflow from the domain outer surface removes the negative pressure and hence increases $\sigma_{m}$ near the boundary. This gives initiation of secondary fractures at the outer boundary. For high $b$, Figure 13 shows that the displacement increases near the borehole when fractures initiate at the borehole, while being almost unchanged near the domain surface. Thus, the tangential stress increases near the borehole. Near the radial midpoint of the fractures seen in the high $b$ case in Figure 16, the pressure $p_{\text {frac }}$ is negative, while $\sigma_{m}$ is of course zero. Thus, the total tangential stress is given by $\sigma_{\theta}=-b p_{\text {frac }}$. Between the fractures, the fluid pressure $p_{m}$ is closer to zero, thus giving $\sigma_{\theta}=\sigma_{m, \theta}-b p_{m}$. To keep $\sigma_{\theta}$ continuous, the 2 expressions for $\sigma_{\theta}$ must be identical, and thus $\sigma_{\theta}=b\left(p_{m}-p_{f r a c}\right)$. Secondary fractures will initiate between the primary fractures when this expression exceeds the tensile strength of the material.

\section{2 | Borehole breakdown}

When hydraulic fracturing tests are conducted to determine the in situ stress in a rock mass, a section of a vertical borehole is pressurized, and 2 vertical fractures perpendicular to the minimum compressive stress are usually initiated. ${ }^{75}$ The pressure development in time is assumed to provide an estimate of the in situ stress. Of particular importance is the peak pressure, which indicates fracture initiation at the borehole wall. This pressure is related to the far-field stress using breakdown criteria. ${ }^{76,77}$ Although these criteria suggest a breakdown pressure that is independent of the injection rate, experimental results suggest an injection rate dependence. ${ }^{78,79}$

This dependence has been attributed to the existence of microcracks in an impermeable rock, ${ }^{80}$ while it has also been argued that compressibility of the injection system and viscous fluid flow could diminish the accuracy of stress estimates. ${ }^{81}$ The results in Section 3.5 further show that in permeable rocks, the injection rate and the Biot coefficient affects the breakdown pressure even without any initial defects. We infer that the injection rate dependence seen in this work is caused by 2 different effects. Firstly, for a slow injection, the pressure diffuses further away from the borehole and contributes to the deformation. Higher pressure is needed to initiate fractures when the pressure is applied in the borehole only. This is seen in Figure 18, where we can notice that for low injection rates, the breakdown pressure is highest for a low Biot coefficient. Secondly, rapid injection causes a negative fluid pressure some distance from the borehole through a Mandel-Cryer effect. This negative pressure prevents a large deformation, and thus means that a higher borehole pressure is required before fractures initiate. The Mandel-Cryer effect is most significant for a high Biot coefficient, and thus, the breakdown pressure for a high injection rate is highest for high $b$.

The second effect discussed above requires a negative fluid pressure. Clearly, any pressure below the initial pressure in the rock is physically meaningless, and thus, the fluid will cavitate in the pores at a given pressure. This limits the 
Mandel-Cryer effect and might reduce the breakdown pressure at a high injection rate. A similar conclusion has also been reached in the impermeable case. ${ }^{80}$

\subsection{Model limitations and further work}

In this work, volume flow in a fracture per unit length in the third dimension is given by a Darcy expression

$$
Q_{D}=\frac{k_{f} A}{\mu} \frac{\Delta p}{d}
$$

where $k_{f}$ is the permeability in the fracture, $A$ is the length of the facet between 2 triangles, $\mu$ is the fluid viscosity, $\Delta p$ is the pressure difference between 2 triangles, and $d$ is the length of the Voronoi segment connecting the 2 triangles (see Figure 4). Another option is to assume that each Voronoi segment represents a straight flow channel bounded by 2 flat plates separated by the particle separation $w .^{82}$ In the fully developed case, this gives a Poiseuille flow between 2 triangles with a volume flow given by the cubic law

$$
Q_{P}=\frac{w^{3}}{12 \mu} \frac{\Delta p}{d}
$$

Setting the 2 expressions for volume flow equal, we get

$$
w=\left(12 k_{f} A\right)^{1 / 3} .
$$

With the grid resolution in this paper, the low fracture permeability $k_{f}=10^{-14} \mathrm{~m}^{2}$ corresponds to $w \approx 10^{-5} \mathrm{~m}$. Clearly, ignoring the accelerated fluid transport in fractures is valid only in very narrow fractures, even for the high-permeability rock studied in part of this work. On the other hand, the high fracture permeability $k_{f}=10^{-11} \mathrm{~m}^{2}$ corresponds to $w \approx 10^{-4} \mathrm{~m}$. This is a good approximation for the fracture aperture that is usually seen in the simulations at early stages of the injection and thus gives a reasonable permeability. However, as the fractures grow towards the domain boundary, the fracture aperture increases, and the fracture permeability becomes an underestimate. A realistic fracture permeability would reduce the borehole pressure in the final state and likely reduce the density of fractures formed late in the injection process.

Although the simplified model for permeability increase is inaccurate, this first approximation is intended to provide some insight into the effect of higher permeability in fractures without going into details in models for fluid flow. A Poiseuille-type flow has been tested in the present model, but for a small aperture fracture, it is often seen that DEM particles are almost in contact in some areas. This causes a low-permeability obstacle to the flow, and thus locally a sharp pressure gradient. Zero-aperture points are also seen in fractures in nature, especially in fractures under compression, but in a real 3D setting, the fluid will likely bypass such obstacles by flowing in the third dimension. Thus, even though Okamoto and Shimizu ${ }^{82}$ treated the local fracture clogging seen in 2D as real, we assume that this effect is nonphysical. Fluid flow models that avoid this problem and provide a realistic flow rate in fractures are left as a topic for further work.

We assume that the material is situated in an isotropic compressive stress field with a magnitude that exactly matches the the stress contribution from the initial fluid pressure $p_{0}$, which makes the material initially stress free. This is the same approach as previously followed by Grassl et al. ${ }^{26}$ However, in natural settings, the stress state might be more complicated. Firstly, the compressive stress might be too high to be canceled by the fluid pressure, causing a compressive stress in the material initially. Furthermore, the external stress field might be anisotropic. A natural extension of the work presented in this paper would be to include a confining pressure and introduce a stress anisotropy. Another highly related issue is the capturing of material anisotropy of geological materials. Materials that are highly relevant for geothermal applications, $\mathrm{CO}_{2}$ storage, hydraulic fractures, and nuclear waste disposal, such as shales, mudstone, and salt, are often inherently anisotropic. ${ }^{83,84}$ As a result, an extension that captures the effects of inherent material anisotropy on the coupling mechanisms between damage and plastic responses during the injection and the potential brittle-ductile transition ${ }^{85,86}$ could be invaluable. These important topics are nevertheless considered to be outside the scope of the present paper and left as a topic for further work. 


\section{5 | CONCLUSION}

A two-way iterative coupling algorithm specific for discrete hydromechanical models has been described. By introducing a proper iteration mechanism between the solid discrete element solver and a fluid flow network model in the transient regime, the proposed model is able to capture the Mandel-Cryer effect and replicate the fluid-driven fractures that occur in a wide spectrum of regimes. Grid refinement studies show that above a minimum threshold the model is insensitive to changes in resolution. Benchmarking against an analytical solution and comparison with 1D results both show that the model performs reasonably well and converges using both drained and undrained operator splits. However, the undrained operator split applied in the discrete hydromechanics model shows a significantly better convergence rate.

Our borehole injection simulations were focused on continuous fluid injection in fluid-saturated permeable rock. Not surprisingly, the resulting fracture pattern is heavily dependent on injection rate and flow rate through the porous media, with high fracture density for high injection rates in low-permeability rock, and less or even no fractures for low injection rates in high-permeability rock. Additionally, the simulations highlight the importance of how efficiently fluid flows through fractures compared to through the bulk porous media, with less intense fracture formation when fluid is allowed to escape rapidly through the fractures. When borehole injection is used to determine the in situ stress in a permeable rock mass, our results clearly show that the injection rate and Biot coefficient has a significant effect on the recorded borehole breakdown pressure. This must be taken into account when the in situ stress is evaluated. We have shown that during borehole injection and fracture formation, the pressure field is far from equilibrium. Especially for low-permeability rock, the pressure field in a large part of the computational domain is mostly unaffected by the fluid injection when the fracture pattern is fully developed and the fluid is efficiently removed at realistic flow rates in the fractures.

\section{ACKNOWLEDGEMENTS}

The first author is supported by project grant 231621 from the Norwegian Research Council and a travel grant from the UiO Energy research initiative, while the second author is supported by the Earth Materials and Processes program from the US Army Research Office under grant contract W911NF-15-1-0442 and W911NF-15-1-0581, the Dynamic Materials and Interactions Program from the Air Force Office of Scientific Research under grant contract FA9550-17-1-0169, the Nuclear Energy University Program from Department of Energy under grant contract DE-NE0008534, and the Earth Science Program under the grant contract EAR-1520732. These supports are gratefully acknowledged. We also thank the 2 anonymous reviewers for their constructive suggestions and feedback.

The views and conclusions contained in this document are those of the authors and should not be interpreted as representing the official policies, either expressed or implied, of the sponsors, including the Army Research Laboratory or the US Government. The US Government is authorized to reproduce and distribute reprints for Government purposes notwithstanding any copyright notation herein.

\section{ORCID}

Ole Ivar Ulven (10) http://orcid.org/0000-0001-7646-0482

\section{REFERENCES}

1. Rice JR, Cleary MP. Some basic stress diffusion solutions for fluid-saturated elastic porous media with compressible constituents. Rev Geophys Space Phys. 1976;14(2):227-241.

2. Rudnicki JW. Fluid mass sources and point forces in linear elastic diffusive solids. Mech Mater. 1986;5(4):383-393.

3. Wang H. Theory of Linear Poroelasticity with Applications to Geomechanics and Hydrogeology. Princeton, New Jersey: Princeton University Press; 2000.

4. Rethore J, Borst Rd, Abellan MA. A two-scale approach for fluid flow in fractured porous media. Int J Numer Methods Eng. 2007;71(7):780-800.

5. Mohammadnejad T, Khoei AR. An extended finite element method for hydraulic fracture propagation in deformable porous media with the cohesive crack model. Finite Elem Anal Des. 2013;73:77-95.

6. Salimzadeh S, Khalili N. A three-phase XFEM model for hydraulic fracturing with cohesive crack propagation. Comput Geotech. 2015;69:82-92.

7. Pouya A. A finite element method for modeling coupled flow and deformation in porous fractured media. Int J Numer Anal Methods Geomech. 2015;39(16):1836-1852. 
8. Gupta P, Duarte CA. Coupled formulation and algorithms for the simulation of non-planar three-dimensional hydraulic fractures using the generalized finite element method. Int J Numer Anal Methods Geomech. 2016;40(10):1402-1437.

9. Mohammadnejad T, Andrade JE. Numerical modeling of hydraulic fracture propagation, closure and reopening using xfem with application to in-situ stress estimation. Int J Numer Anal Methods Geomech. 2016;40(15):2033-2060.

10. Samimi S, Pak A. A fully coupled element-free galerkin model for hydro-mechanical analysis of advancement of fluid-driven fractures in porous media. Int J Numer Anal Methods Geomech. 2016;40(16):2178-2206.

11. Settgast RR, Fu P, Walsh SD, White JA, Annavarapu C, Ryerson FJ. A fully coupled method for massively parallel simulation of hydraulically driven fractures in 3-dimensions. Int J Numer Anal Methods Geomech. 2017;41(5):627-653.

12. Liu F, Gordon P, Meier H, Valiveti D. A stabilized extended finite element framework for hydraulic fracturing simulations. Int J Numer Anal Methods Geomech. 2017;41(5):654-681.

13. Wick T, Singh G, Wheeler MF. Pressurized-fracture propagation using a phase-field approach coupled to a reservoir simulator. Society of Petroleum Engineers; 2014. https://doi.org/10.2118/168597-MS

14. Mikelic A, Wheeler MF, Wick T. A phase-field method for propagating fluid-filled fractures coupled to a surrounding porous medium. Multiscale Model Simul. 2015;13(1):367-398.

15. Heister T, Wheeler MF, Wick T. A primal-dual active set method and predictor-corrector mesh adaptivity for computing fracture propagation using a phase-field approach. Comput Meth Appl Mech Eng. 2015;290:466-495.

16. Miehe $\mathrm{C}$, Mauthe $\mathrm{S}$, Teichtmeister S. Minimization principles for the coupled problem of Darcy-Biot-type fluid transport in porous media linked to phase field modeling of fracture. J Mech Phys Solids. 2015;82:186:217.

17. Ingraffea A, Saouma V. Numerical modeling of discrete crack propagation in reinforced and plain concrete. In: Sih GC, DiTommaso, A. (eds) Fracture Mechanics of Concrete: Structural Application and Numerical Calculation. Springer Dordrecht; 1985:171-225.

18. Hunsweck MJ, Shen Y, Lew AJ. A finite element approach to the simulation of hydraulic fractures with lag. Int J Numer Anal Methods Geomech. 2013;37(9):993-1015.

19. Meschke G, Leonhart D. A generalized finite element method for hydro-mechanically coupled analysis of hydraulic fracturing problems using space-time variant enrichment functions. Comput Meth Appl Mech Eng. 2015;290:438-465.

20. Mota A, Knap J, Ortiz M. Fracture and fragmentation of simplicial finite element meshes using graphs. Int J Numer Methods Eng. 2008;73(11):1547-1570.

21. Wriggers P. Nonlinear Finite Element Methods. Springer-Verlag Berlin Heidelberg; 2008.

22. Mota A, Sun W, Ostien JT, Foulk Iii JW, Long KN. Lie-group interpolation and variational recovery for internal variables. Comput Mech. 2013;52(6):1281-1299.

23. Wheeler M, Wick T, Wollner W. An augmented-Lagrangian method for the phase-field approach for pressurized fractures. Comput Meth Appl Mech Eng. 2014;271:69-85.

24. Cundall PA, Strack OD. A discrete numerical model for granular assemblies. Geotechnique. 1979;29(1):47-65.

25. Schauffert EA, Cusatis G. Lattice discrete particle model for fiber-reinforced concrete. I: Theory. J Eng Mech. 2011;138(7):826-833.

26. Grassl P, Fahy C, Gallipoli D, Wheeler S. J. On a 2D hydro-mechanical lattice approach for modelling hydraulic fracture. J Mech Phys Solids. 2015;75:104-118.

27. Yao C, Jiang QH, Shao JF. Numerical simulation of damage and failure in brittle rocks using a modified rigid block spring method. Comput Geotech. 2015;64:48-60.

28. Jiao YY, Zhang HQ, Zhang XL, Li HB, Jiang QH. A two-dimensional coupled hydromechanical discontinuum model for simulating rock hydraulic fracturing. Int J Numer Anal Methods Geomech. 2015;39(5):457-481.

29. Verde A, Ghassemi A. Fast multipole displacement discontinuity method (fm-ddm) for geomechanics reservoir simulations. Int J Numer Anal Methods Geomech. 2015;39(18):1953-1974.

30. Zhang F, Dontsov E, Mack M. Fully coupled simulation of a hydraulic fracture interacting with natural fractures with a hybrid discrete-continuum method. Int J Numer Anal Methods Geomech. 2017;41(13):1430-1452.

31. Røyne A, Jamtveit Bjørn, Mathiesen J, Malthe-Sørenssen A. Controls on rock weathering rates by reaction-induced hierarchical fracturing. Earth Planet Sci Lett. 2008;275(3-4):364-369.

32. Ulven O. I, Storheim H, Austrheim H, Malthe-Sørenssen A. Fracture initiation during volume increasing reactions in rocks and applications for $\mathrm{CO}_{2}$ sequestration. Earth Planet Sci Lett. 2014;389:132-142.

33. Ulven O. I, Jamtveit B, Malthe-Sørenssen A. Reaction-driven fracturing of porous rock. J Geophys Res Solid Earth. 2014;119(10):7473-7486.

34. Satake M. Constitution of mechanics of granular materials through the graph theory. In Cowin SC, Satake, M. (eds), Continuum Mech Stat Approaches Mech Granular Mater. Gabujutsa Bunfer Fukyukaii, Tokyo; 1978:47-62.

35. Satake M. A discrete-mechanical approach to granular materials. Int J Eng Sci. 1992;30(10):1525-1533.

36. Wang K, Sun W. Anisotropy of a tensorial bishop's coefficient for wetted granular materials. J Eng Mech. 2015;143(3), B4015004.

37. Kuhn M. R, Sun WaiChing, Wang Q. Stress-induced anisotropy in granular materials: fabric, stiffness, and permeability. Acta Geotech. 2015:1-21.

38. Biot M. General theory of three-dimensional consolidation. J Appl Phys. 1941;12:155-164.

39. Coussy O. Mechanics and Physics of Porous Solids. Chichester, West Sussex: Wiley; 2010.

40. Ulven OI, Beinlich A, Hövelmann J, Austrheim H, Jamtveit B. Subarctic physicochemical weathering of serpentinized peridotite. Earth Planet Sci Lett. 2017;468:11-26. 
41. Schlangen E, Van Mier J. Simple lattice model for numerical simulation of fracture of concrete materials and structures. Mater Struct. 1992;25(9):534-542.

42. Schlangen E, Garboczi E. Fracture simulations of concrete using lattice models: computational aspects. Eng Fract Mech. 1997;57(2):319-332.

43. Bolander JE, Berton S. Simulation of shrinkage induced cracking in cement composite overlays. Cem Concr Compos. 2004;26(7):861-871.

44. Roux S. 3 - continuum and discrete description of elasticity and other rheological behaviour. In: Herrmann HJ, Roux S, eds. Statistical Models for the Fracture of Disordered Media, Random Materials and Processes. Amsterdam: North-Holland; 1990:87-114.

45. Lilliu G, van Mier JG. 3D lattice type fracture model for concrete. Eng Fract Mech. 2003;70(7):927-941.

46. Mosler J, Meschke G. Embedded crack vs. smeared crack models: a comparison of elementwise discontinuous crack path approaches with emphasis on mesh bias. Comput Meth Appl Mech Eng. 2004;193(30):3351-3375.

47. Yip M, Li Z, Liao BS, Bolander J. Irregular lattice models of fracture of multiphase particulate materials. Int J Fract. 2006;140(1-4):113-124.

48. Leon SE, Spring DW, Paulino GH. Reduction in mesh bias for dynamic fracture using adaptive splitting of polygonal finite elements. Int J Numer Methods Eng. 2014;100(8):555-576.

49. Grassl P, Jirásek M. Meso-scale approach to modelling the fracture process zone of concrete subjected to uniaxial tension. Int $J$ Solids Struct. 2010;47(7):957-968.

50. Kuleci H, Ulven OI, Rybacki E, Wunder B, Abart R. Reaction-induced fracturing in a hot pressed calcite-periclase aggregate. J Struct Geol. 2017;94(Supplement C):116-135.

51. Song JH, Wang H, Belytschko T. A comparative study on finite element methods for dynamic fracture. Comput Mech. 2008;42(2):239-250.

52. Wang K, Sun W. A unified variational eigen-erosion framework for interacting brittle fractures and compaction bands in fluid-infiltrating porous media. Comput Meth Appl Mech Eng. 2017;318:1-32.

53. Tildesley D, Allen M. Computer Simulations of Liquids. Oxford, U. K.: Clarendon; 1987.

54. Pollard DD, Fletcher RC. Fundamentals of Structural Geology. Cambridge: Cambridge University Press; 2005.

55. Anderson TL. Fracture Mechanics : Fundamentals and Applications. 3rd ed. Boca Raton, FL: Taylor \& Francis; 2005.

56. Coussy O. Poromechanics. Chichester, West Sussex: John Wiley \& Sons; 2004.

57. Prevost JH. Nonlinear transient phenomena in saturated porous media. Comput Meth Appl Mech Eng. 1982;30(1):3-18.

58. Borja RI. One-step and linear multistep methods for nonlinear consolidation. Comput Meth Appl Mech Eng. 1991;85(3):239-272.

59. Zienkiewicz OC, Chan A, Pastor M, Schrefler B, Shiomi T. Computational Geomechanics; Wiley Chichester; 1999.

60. Sun W, Ostien JT, Salinger AG. A stabilized assumed deformation gradient finite element formulation for strongly coupled poromechanical simulations at finite strain. Int J Numer Anal Methods Geomech. 2013;37(16):2755-2788.

61. Sun W, Chen Q, Ostien JT. Modeling the hydro-mechanical responses of strip and circular punch loadings on water-saturated collapsible geomaterials. Acta Geotech. 2014;9(5):903-934.

62. Sun W. A stabilized finite element formulation for monolithic thermo-hydro-mechanical simulations at finite strain. Int J Numer Methods Eng. 2015;103(11):798-839.

63. Armero F. Formulation and finite element implementation of a multiplicative model of coupled poro-plasticity at finite strains under fully saturated conditions. Comput Meth Appl Mech Eng. 1999;171(3):205-241.

64. Markert B, Heider Y, Ehlers W. Comparison of monolithic and splitting solution schemes for dynamic porous media problems. Int J Numer Methods Eng. 2010;82(11):1341-1383.

65. Jha B, Juanes R. Coupled multiphase flow and poromechanics: a computational model of pore pressure effects on fault slip and earthquake triggering. Water Resour Res. 2014;50(5):3776-3808.

66. Castelletto N, White J, Tchelepi H. Accuracy and convergence properties of the fixed-stress iterative solution of two-way coupled poromechanics. Int J Numer Anal Methods Geomech. 2015;39(14):1593-1618.

67. Tordesillas A, Walker DM, Lin Q. Force cycles and force chains. Phys Rev E. 2010;81(1):011 302.

68. Detournay E. Mechanics of hydraulic fractures. Annu Rev Fluid Mech. 2016;48:311-339.

69. Chau KT. Fluid point source and point forces in linear elastic diffusive half-spaces. Mech Mater. 1996;23(3):241-253.

70. Jeremić B, Cheng Z, Taiebat M, Dafalias Y. Numerical simulation of fully saturated porous materials. Int J Numer Anal Methods Geomech. 2008;32(13):1635-1660.

71. Wang K, Sun W. A semi-implicit discrete-continuum coupling method for porous media based on the effective stress principle at finite strain. Comput Meth Appl Mech Eng. 2016;304:546-583.

72. Mandel J. Consolidation des sols (étude mathématique)*. Geotechnique. 1953;3(7):287-299.

73. Cryer CW. A comparison of the three-dimensional consolidation theories of Biot and Terzaghi. Q J Mech Appl Math. 1963;16(4):401-412.

74. Uzuoka R, Borja RI. Dynamics of unsaturated poroelastic solids at finite strain. Int J Numer Anal Methods Geomech. 2012;36(13):1535-1573.

75. Haimson BC. The hydrofracturing stress measuring method and recent field results. Int J Rock Mech Min Sci Geomech Abstr. 1978;15(4):167-178.

76. Hubbert MK, Willis D. G. Mechanics of Hydraulic Fracturing. Society of Petroleum Engineering of AIME; 1959;2(10):153-168.

77. Haimson B, Fairhurst C. Initiation and extension of hydraulic fractures in rocks. Soc Pet Eng. 1967;7(03):310-318.

78. Schmitt DR, Zoback MD. Diminished pore pressure in low-porosity crystalline rock under tensional failure: apparent strengthening by dilatancy. J Geophys Res Solid Earth. 1992;97(B1):273-288. 
79. Schmitt DR, Zoback MD. Infiltration effects in the tensile rupture of thin walled cylinders of glass and granite: implications for the hydraulic fracturing breakdown equation. Int J Rock Mech Min Sci Geomech Abstr. 1993;30(3):289-303.

80. Garagash D, Detournay E. An analysis of the influence of the pressurization rate on the borehole breakdown pressure. Int J Solids Struct. 1997;34(24):3099-3118.

81. Lakirouhani A, Detournay E, Bunger AP. A reassessment of in situ stress determination by hydraulic fracturing. Geophys $J$ Int. 2016;205(3):1859-1873.

82. Okamoto A, Shimizu H. Contrasting fracture patterns induced by volume-increasing and -decreasing reactions: implications for the progress of metamorphic reactions. Earth Planet Sci Lett. 2015;417:9-18.

83. Semnani SJ, White JA, Borja RI. Thermoplasticity and strain localization in transversely isotropic materials based on anisotropic critical state plasticity. Int J Numer Anal Methods Geomech. 2016;40(18):2423-2449.

84. Na SH, Sun WC, Ingraham MD, Yoon H. Effects of spatial heterogeneity and material anisotropy on the fracture pattern and macroscopic effective toughness of mancos shale in brazilian tests. J Geophys Res Solid Earth. 2017;122(8):6202-6230.

85. Tjioe M, Borja RI. On the pore-scale mechanisms leading to brittle and ductile deformation behavior of crystalline rocks. Int J Numer Anal Methods Geomech. 2015;39(11):1165-1187.

86. Choo J, Sun W. Coupled phase-field and plasticity modeling of geological materials: from brittle fracture to ductile flow. Comput Meth Appl Mech Eng. 2017;330:1-32.

87. Sneddon IN. The distribution of stress in the neighbourhood of a crack in an elastic solid. Proc R Soc London A: Math Phys Eng Sci. 1946;187(1009):229-260.

How to cite this article: Ulven OI, Sun W. Capturing the two-way hydromechanical coupling effect on fluid-driven fracture in a dual-graph lattice beam model. Int J Numer Anal Methods Geomech. 2017;1-32. https://doi.org/10.1002/nag.2763

\section{APPENDIX A: THE 1D FINITE DIFFERENCE MODEL}

The finite difference method is a simplified 1D continuum based model that assumes radial symmetry in a domain containing a borehole under plane strain conditions. With these assumptions, the equations of linear elasticity for the displacement $u$ become

$$
\frac{\mathrm{d}^{2} u}{\mathrm{~d} r^{2}}+\frac{1}{r} \frac{\mathrm{d} u}{\mathrm{~d} r}-\frac{u}{r^{2}}=\frac{b(1+v)(1-2 v)}{E(1-v)} \frac{\mathrm{d} p}{\mathrm{~d} r}
$$

where $r$ denotes radial position, $b$ is Biot coefficient, $v$ is Poisson ratio, and $E$ is Young modulus. The right hand side term stems from the $\sigma_{f}$ part of Equation 1. Fluid transport is again described in dimensionless form by Equation 14, repeated here for reference without the source term:

$$
\frac{E}{M} \int_{V^{*}} \frac{\partial p^{*}}{\partial t^{*}} \mathrm{~d} V^{*}-\int_{S^{*}} \nabla^{*} p^{*} \cdot \mathbf{n} \mathrm{d} S^{*}+b \int_{V^{*}} \frac{\partial \varepsilon_{v}}{\partial t^{*}} \mathrm{~d} V^{*}=0
$$

where again $V^{*}$ is the volume Equation 2 is integrated over, $S^{*}$ denotes the surface of $V^{*}, \mathbf{n}$ is an outwards pointing unit vector from the surface $S^{*}, M$ denotes the Biot modulus, $p^{*}$ is pressure, $t^{*}$ is time, $k$ is permeability, $\mu$ is fluid viscosity, and $\varepsilon_{v}$ denotes volumetric strain. We now write Equation A1 on dimensionless form by introducing the dimensionless variables $u^{*}=\frac{u}{R}$ and $r^{*}=\frac{r}{R}$, where $R$ is again the domain radius. This leads to the dimensionless equation

$$
\frac{\mathrm{d}^{2} u^{*}}{\mathrm{~d} r^{* 2}}+\frac{1}{r^{*}} \frac{\mathrm{d} u^{*}}{\mathrm{~d} r^{*}}-\frac{u^{*}}{r^{* 2}}=\frac{b(1+v)(1-2 v)}{(1-v)} \frac{\mathrm{d} p^{*}}{\mathrm{~d} r^{*}}
$$

Equation A3 is valid until the first fracture is formed, thus breaking the radial symmetry. This limits the 1D model to studies of prebreaking dynamics and to determining the fracture mode and the location of the first fracture that might form.

For simplicity, we from here on skip the asterisk $\left(^{*}\right)$ on dimensionless variables. Equation A3 is discretized using a centered difference scheme, which gives 


$$
\begin{aligned}
u_{i+1}^{n+1}\left(\frac{1}{\Delta r^{2}}+\frac{1}{2 r_{i} \Delta r}\right) & -u_{i}^{n+1}\left(\frac{2}{\Delta r^{2}}+\frac{1}{r_{i}^{2}}\right)+u_{i-1}^{n+1}\left(\frac{1}{\Delta r^{2}}-\frac{1}{2 r_{i} \Delta r}\right) \\
& -\left(p_{i+1}^{n+1}-p_{i-1}^{n+1}\right) \frac{b(v+1)(2 v-1)}{2(v-1) \Delta r}=0,
\end{aligned}
$$

where $i=0 \ldots, n_{r}$ denotes points in the spatial discretization and $n$ denotes time steps in the temporal discretization. The integration volumes in Equation A2 are assumed to be rings of approximate volume $V_{i}=2 \pi r_{i} \Delta r$ with surfaces $S_{i}^{+}=2 \pi\left(r_{i}+\Delta r / 2\right), S_{i}^{-}=2 \pi\left(r_{i}-\Delta r / 2\right)$. Thus, the surface integral in Equation A4 simplifies to a sum with only 2 terms: the flux in negative direction through the surface $S_{i}^{-}$and the flux in positive direction through the surface $S_{i}^{+}$. The volumetric strain is given by

$$
\varepsilon_{v}=\frac{1}{3}\left(\varepsilon_{r}+\varepsilon_{\theta}\right)=\frac{1}{3}\left(\frac{\mathrm{d} u}{\mathrm{~d} r}+\frac{u}{r}\right) \approx \frac{u_{i+1}^{n+1}}{6 \Delta r}+\frac{u_{i}^{n+1}}{3 r_{i}}-\frac{u_{i-1}^{n+1}}{6 \Delta r}
$$

where $\varepsilon_{r}, \varepsilon_{\theta}$ denote radial and tangential strain, respectively. This leads to the discretized equation for pressure evolution

$$
\begin{aligned}
& -p_{i+1}^{n+1} \frac{\Delta t}{\Delta r}\left(\frac{1}{\Delta r}+\frac{1}{2 r_{i}}\right)+p_{i}^{n+1}\left(\frac{1}{M}+\frac{2 \Delta t}{\Delta r^{2}}\right)-p_{i-1}^{n+1} \frac{\Delta t}{\Delta r}\left(\frac{1}{\Delta r}-\frac{1}{2 r_{i}}\right) \\
& +u_{i+1}^{n+1} \frac{b}{6 \Delta r}+u_{i}^{n+1} \frac{b}{3 r_{i}}-u_{i-1}^{n+1} \frac{b}{6 \Delta r}=u_{i+1}^{n} \frac{b}{6 \Delta r}+u_{i}^{n} \frac{b}{3 r_{i}}-u_{i-1}^{n} \frac{b}{6 \Delta r}+p_{i}^{n} \frac{1}{M}
\end{aligned}
$$

The boundary conditions are

- No flux in negative direction at $i=0$.

- Source term of magnitude $Q / 2 \pi$ at $i=0$.

- Constant pressure $p_{s}=0$ at outer boundary.

- $\sigma_{m r}=-(1-b) p_{0}^{n+1}$ at $i=0$.

- $\sigma_{m r}=-(1-b) p_{s}=0$ at $i=n_{r}$.

Here, $\sigma_{m r}$ denotes the dimensionless radial component of the effective stress.

The initial conditions are zero pressure and displacement. The full system defined by Equations A4 and A6 with specified boundary conditions and initial conditions is solved monolithically.

\section{APPENDIX B: ANALYTICAL SOLUTIONS FOR STEADY STATE}

Grassl et $\mathrm{al}^{26}$ derived the pressure distribution and displacement for a borehole injection assuming plane stress. In this section, we redo a similar derivation, with the assumption of plane strain.

With steady state fluid injection in a radial symmetry, the fluid pressure Equation 2 on dimensionless form simplifies to

$$
\frac{1}{r^{*}} \frac{\partial}{\partial r^{*}}\left(r^{*} \frac{\partial p^{*}}{\partial r^{*}}\right)=0
$$

The displacement is still controlled by Equation A3, repeated here for reference:

$$
\frac{\mathrm{d}^{2} u^{*}}{\mathrm{~d} r^{* 2}}+\frac{1}{r^{*}} \frac{\mathrm{d} u^{*}}{\mathrm{~d} r^{*}}-\frac{u^{*}}{r^{* 2}}=\frac{b(1+v)(1-2 v)}{(1-v)} \frac{\mathrm{d} p^{*}}{\mathrm{~d} r^{*}}
$$

Grassl et $\mathrm{al}^{26}$ provided the solution to Equation B1, which is, when given in the present dimensionless formulation, given by 


$$
p^{*}=-\frac{Q}{2 \pi} \ln r^{*}
$$

It directly follows that the dimensionless pressure in the borehole at steady state is $p_{h}^{*}=-\frac{Q}{2 \pi} \ln R_{i}^{*}$, where $R_{i}^{*}=\frac{R_{i}}{R}$ and $R_{i}$ is the radius of the borehole.

Inserting for pressure from Equation B3 in Equation B2 then gives

$$
\frac{\mathrm{d}^{2} u^{*}}{\mathrm{~d} r^{* 2}}+\frac{1}{r^{*}} \frac{\mathrm{d} u^{*}}{\mathrm{~d} r^{*}}-\frac{u^{*}}{r^{* 2}}+\frac{b Q(1+v)(1-2 v)}{2 \pi(1-v)} \frac{1}{r^{*}}=0
$$

which differs from the equation presented by Grassl et $\mathrm{a}^{26}$ only in $v$-dependence, sign convention, and dimensionless formulation. Thus, the solution of this differential equation follows the same pattern as the solution in Grassl et $\mathrm{al}^{26}$ :

$$
u^{*}=-\frac{\lambda b p_{h}^{*}}{2(1-v)} \frac{r^{*} \ln r^{*}}{\ln R_{i}^{*}}+\frac{C_{1}}{r^{*}}+C_{2} r^{*}
$$

where for convenience the dimensionless factor $\lambda=(1+v)(1-2 v)$ has been introduced and $C_{1}, C_{2}$ are integration constants. The constants can be determined by requiring that $\left.\sigma_{m r}^{*}\right|_{r^{*}=R_{i}^{*}}=(1-b) p_{h}^{*}$ and $\left.\sigma_{m r}^{*}\right|_{r^{*}=1}=0$, where $\sigma_{m r}^{*}$ is the radial component of the effective stress divided by $E$. It follows that the displacement is given by

$$
\begin{aligned}
u^{*}= & \frac{b p_{h}^{*}}{2}\left(\frac{\lambda r^{*}}{\ln R_{i}^{*}}\left((1-v) \ln r^{*}-1\right)-\frac{R_{i}^{* 2}}{1-R_{i}^{* 2}} \frac{1-2 v}{1-v}\left(\frac{1+v}{r^{*}}+\lambda r^{*}\right)\right) \\
& +p_{h}^{*} \frac{R_{i}^{* 2}}{1-R_{i}^{* 2}}\left(\frac{1+v}{r^{*}}+\lambda r^{*}\right),
\end{aligned}
$$

and the effective stress components are given by

$$
\begin{aligned}
\sigma_{m r}^{*}= & \frac{b p_{h}^{*}}{2}\left(\frac{\ln r^{*}}{(1-v) \ln R_{i}^{*}}+\frac{R_{i}^{* 2}}{1-R_{i}^{* 2}} \frac{1-2 v}{1-v}\left(\frac{1}{r^{* 2}}-1\right)\right) \\
& +p_{h}^{*} \frac{R_{i}^{* 2}}{1-R_{i}^{* 2}}\left(1-\frac{1}{r^{* 2}}\right) \\
\sigma_{m \theta}^{*}= & \frac{b p_{h}^{*}}{2}\left(\frac{\ln r^{*}-1+2 v}{(1-v) \ln R_{i}^{*}}-\frac{R_{i}^{* 2}}{1-R_{i}^{* 2}} \frac{1-2 v}{1-v}\left(\frac{1}{r^{* 2}}+1\right)\right) \\
+ & p_{h}^{*} \frac{R_{i}^{* 2}}{1-R_{i}^{* 2}}\left(\frac{1}{r^{* 2}}+1\right) \\
\sigma_{m z}^{*}= & b p_{h}^{* v}\left(\frac{\ln r^{*}-1+2 v}{(1-v) \ln R_{i}^{*}}-\frac{R_{i}^{* 2}}{1-R_{i}^{* 2}} \frac{1-2 v}{1-v}\right) \\
& +2 p_{h}^{*} v \frac{R_{i}^{* 2}}{1-R_{i}^{* 2}} .
\end{aligned}
$$

\section{APPENDIX C: THE SNEDDON SOLUTION}

The aperture of a pressurized fracture in 2D plane strain (Figure C1) in an initially unstressed impermeable material has been shown to be elliptic, with an aperture given by ${ }^{87}$ : 


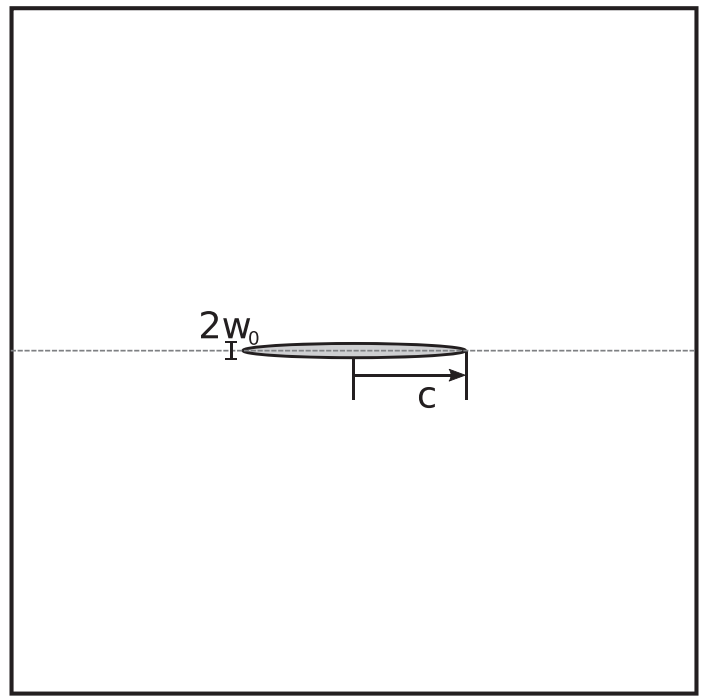

FIGURE C1 Geometry for the Sneddon solution, showing a fracture of length $2 c$ and maximum aperture $2 w_{0}$

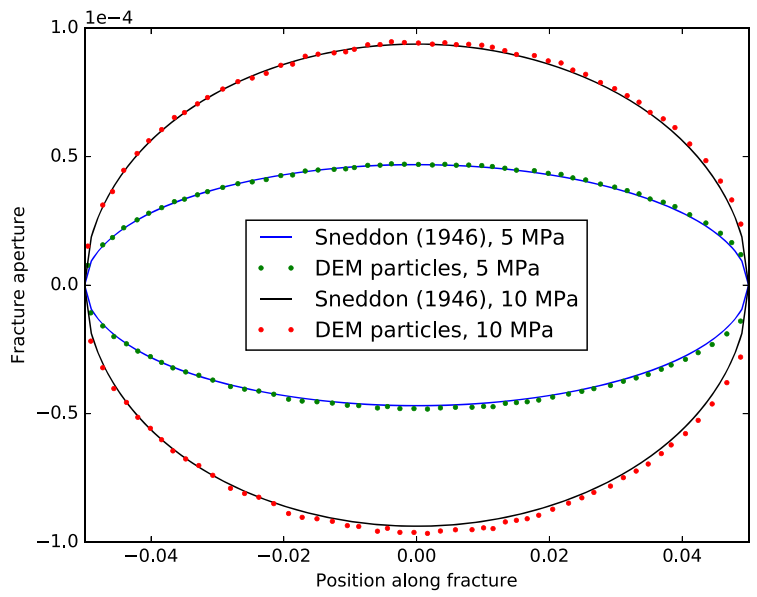

FIGURE C2 Comparison of analytical fracture aperture and particle positions along the simulated fracture [Colour figure can be viewed at wileyonlinelibrary.com]

$$
\left(\frac{x}{c}\right)^{2}+\left(\frac{w}{\varepsilon}\right)^{2}=1,
$$

where $x$ denotes distance along the fracture with $x=0$ in the center of the fracture, $2 c$ is the fracture length, $2 w$ is the fracture aperture, and $\varepsilon=2\left(1-v^{2}\right) p c / E . p$ is the constant pressure in the fracture.

In the discrete element model, the pressurized fracture was implemented by predefining a fracture in a material with zero permeability and defining a constant pressure in the fracture. To avoid significant influence from the finite size of the domain, the fracture length was $10 \%$ of the domain size. Figure $\mathrm{C} 2$ shows comparisons of the analytical solution with positions of discrete element model particles along the fracture in the numerical solution for $p=5 \mathrm{MPa}$ and $p=10 \mathrm{MPa}$. Apart from minor variations caused by the unstructured grid, the numerical simulations fit the analytical solutions well. 